Table S4: Full Gene ontology results from GREAT analysis

From Sawaya et al. PLOS ONE

GREAT Table Output: Genomic Regions Enrichment of Annotations Tool, Bejerano Lab, Stanford University

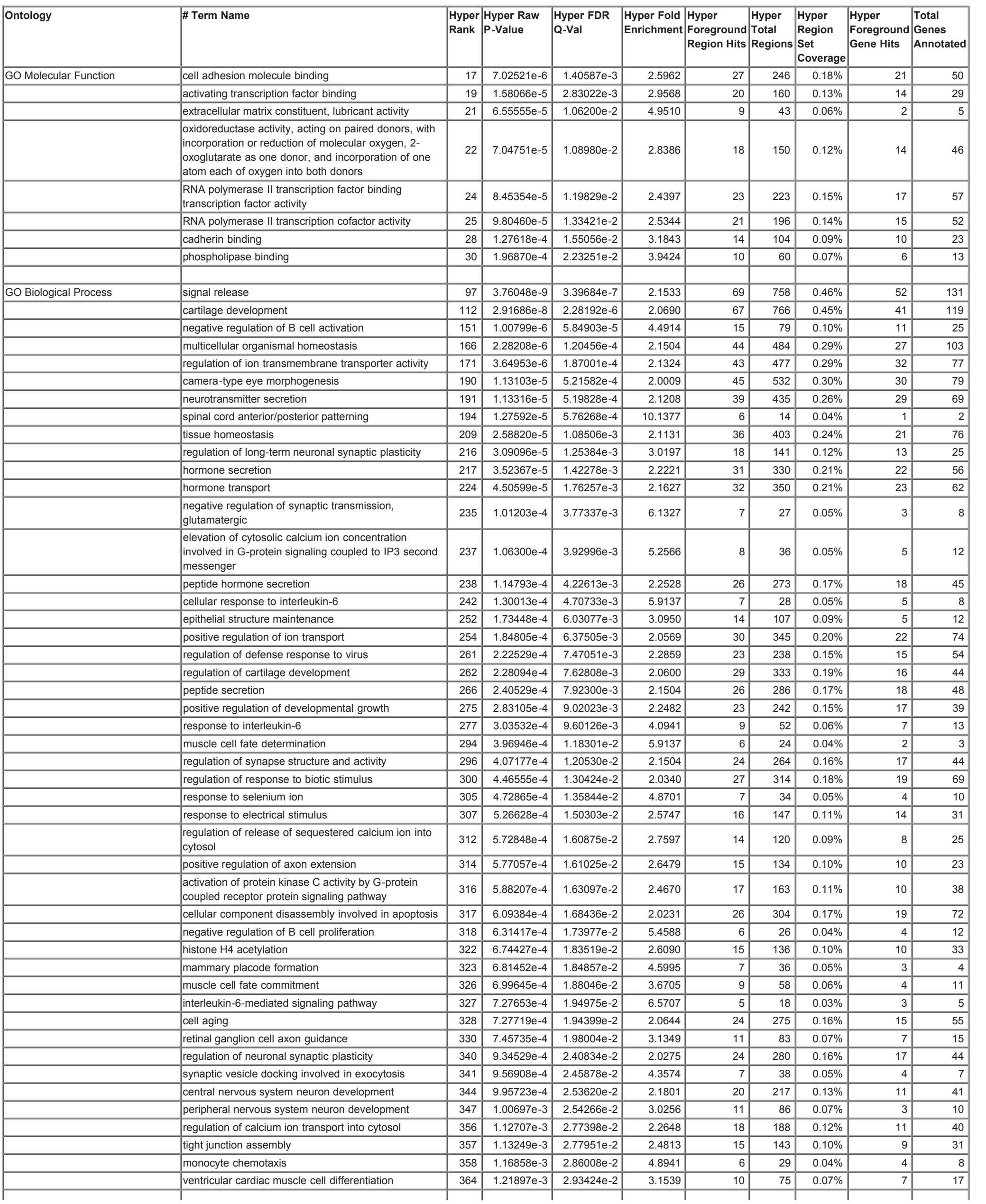


response to cholesterol

\begin{tabular}{|l|l|l|}
369 & $1.27371 \mathrm{e}-3 \mid$ & $3.02445 \mathrm{e}-2 \mid$
\end{tabular}

transcription initiation from RNA polymerase II

promoter

positive regulation of calcium ion transport

spleen development

ectodermal placode formation

retina morphogenesis in camera-type eye

cellular response to lipid

regulation of erythrocyte differentiation

apical junction assembly

regulation of amyloid precursor protein biosynthetic

process

histone ubiquitination

positive regulation of small GTPase mediated signal

transduction

dendrite morphogenesis

protein oxidation

activation of phospholipase D activity by G-protein

coupled receptor protein signaling pathway

protein kinase $\mathrm{C}$ deactivation

prepulse inhibition

regulation of chondrocyte differentiation

negative regulation of transporter activity

negative regulation of ion transmembrane transporter

activity

fat-soluble vitamin metabolic process

angiogenesis involved in wound healing

cell differentiation involved in metanephros

development

distal tubule development

renal system process

cellular response to osmotic stress

negative regulation of insulin secretion

interleukin-12-mediated signaling pathway

myoblast proliferation

glutamate catabolic process

negative regulation of cytokine biosynthetic process

GO Cellular Component

AP-2 adaptor complex

clathrin coat of endocytic vesicle

clathrin sculpted gamma-aminobutyric acid transport

vesicle membrane

inner mucus layer

outer mucus layer

SAGA-type complex

clathrin coat of coated pit

clathrin-coated endocytic vesicle membrane

Mouse Phenotype

abnormal aggression-related behavior

weakness

decreased aggression

decreased aggression towards mice

abnormal heart right ventricle size

abnormal presacral vertebrae

increased lumbar vertebrae number

cervical vertebral transformation

increased presacral vertebrae number

abnormal palatal shelf elevation

increased heart right ventricle size

increased cell proliferation

fused carpal bones

abnormal circulating dihydrotestosterone level

domed cranium

abnormal pituitary gland development

abnormal patella morphology

scaly skin

jerky movement

micrognathia

thin uterus

\begin{tabular}{|c|c|c|c|}
\hline 369 & $1.213 / 1 \mathrm{e}-3$ & $3.02445 \mathrm{e}-2$ & 3.1705 \\
\hline 371 & $1.29986 \mathrm{e}-3$ & $3.06990 \mathrm{e}-2$ & 2.0488 \\
\hline 371 & $1.29986 \mathrm{e}-3$ & $3.06990 \mathrm{e}-2$ & 2.0488 \\
\hline 377 & $1.36187 e-3$ & $3.16517 \mathrm{e}-2$ & 2.5280 \\
\hline 378 & $1.39289 e-3$ & $3.22872 \mathrm{e}-2$ & 2.7559 \\
\hline 381 & $1.40214 \mathrm{e}-3$ & $3.22455 \mathrm{e}-2$ & 2.3508 \\
\hline 384 & $1.46844 \mathrm{e}-3$ & $3.35065 e-2$ & 2.8911 \\
\hline 385 & $1.47193 e-3$ & $3.34989 \mathrm{e}-2$ & 2.2719 \\
\hline 390 & $1.59450 \mathrm{e}-3$ & $3.58232 \mathrm{e}-2$ & 2.3219 \\
\hline 396 & $1.68497 e-3$ & $3.72820 \mathrm{e}-2$ & 4.5783 \\
\hline 401 & $1.75734 \mathrm{e}-3$ & $3.83985 e-2$ & 2.5626 \\
\hline 401 & $1.75734 \mathrm{e}-3$ & $3.83985 e-2$ & 2.5626 \\
\hline 403 & $1.76380 \mathrm{e}-3$ & $3.83484 \mathrm{e}-2$ & 2.2340 \\
\hline 405 & $1.78707 e-3$ & $3.86624 \mathrm{e}-2$ & 23.6546 \\
\hline 405 & $1.78707 e-3$ & $3.86624 e-2$ & 23.6546 \\
\hline 405 & $1.78707 e-3$ & $3.86624 \mathrm{e}-2$ & 23.6546 \\
\hline 408 & $1.79720 \mathrm{e}-3$ & $3.85957 e-2$ & 3.2256 \\
\hline 409 & $1.80599 \mathrm{e}-3$ & $3.86896 e-2$ & 2.0750 \\
\hline 412 & $1.89389 \mathrm{e}-3$ & $4.02774 \mathrm{e}-2$ & 2.5414 \\
\hline 413 & $1.91772 \mathrm{e}-3$ & $4.06853 e-2$ & 2.7979 \\
\hline 416 & $1.92425 \mathrm{e}-3$ & $4.05295 \mathrm{e}-2$ & 2.2800 \\
\hline 417 & $1.94215 e-3$ & $4.08085 e-2$ & 5.3760 \\
\hline 420 & $1.99925 e-3$ & $4.17081 \mathrm{e}-2$ & 3.1775 \\
\hline 421 & $2.00028 \mathrm{e}-3$ & $4.16306 \mathrm{e}-2$ & 4.4352 \\
\hline 422 & $2.00143 e-3$ & $4.15557 \mathrm{e}-2$ & 2.0569 \\
\hline 423 & $2.03250 \mathrm{e}-3$ & $4.21011 \mathrm{e}-2$ & 3.8507 \\
\hline 432 & $2.22020 \mathrm{e}-3$ & $4.50310 \mathrm{e}-2$ & 2.3191 \\
\hline 434 & $2.27112 \mathrm{e}-3$ & $4.58514 \mathrm{e}-2$ & 6.7585 \\
\hline 438 & $2.32522 \mathrm{e}-3$ & $4.65150 \mathrm{e}-2$ & 10.1377 \\
\hline 442 & $2.39588 \mathrm{e}-3$ & $4.74948 e-2$ & 5.1423 \\
\hline 446 & $2.45082 \mathrm{e}-3$ & $4.81481 \mathrm{e}-2$ & 2.2263 \\
\hline 46 & $4.06640 \mathrm{e}-4$ & $9.90963 e-3$ & 3.9424 \\
\hline 55 & $9.00428 \mathrm{e}-4$ & $1.83524 \mathrm{e}-2$ & 3.5482 \\
\hline 59 & $1.11481 \mathrm{e}-3$ & $2.11814 \mathrm{e}-2$ & 3.7847 \\
\hline 63 & $1.55454 \mathrm{e}-3$ & $2.76609 \mathrm{e}-2$ & 5.6320 \\
\hline 63 & $1.55454 \mathrm{e}-3$ & $2.76609 e-2$ & 5.6320 \\
\hline 66 & $1.89389 e-3$ & $3.21675 e-2$ & 2.5414 \\
\hline 70 & $2.21900 \mathrm{e}-3$ & $3.55356 e-2$ & 3.1308 \\
\hline 72 & $2.45752 \mathrm{e}-3$ & $3.82622 \mathrm{e}-2$ & 3.0854 \\
\hline 69 & $3.49871 \mathrm{e}-8$ & $3.69849 \mathrm{e}-6$ & 2.8012 \\
\hline 73 & $9.97917 \mathrm{e}-8$ & $9.97097 e-6$ & 2.1720 \\
\hline 112 & $1.28188 \mathrm{e}-6$ & $8.34824 e-5$ & 3.3564 \\
\hline 116 & $1.52081 \mathrm{e}-6$ & $9.56274 \mathrm{e}-5$ & 4.1138 \\
\hline 122 & $2.18638 \mathrm{e}-6$ & $1.30717 \mathrm{e}-4$ & 2.1332 \\
\hline 127 & $2.64655 e-6$ & $1.52000 \mathrm{e}-4$ & 4.1743 \\
\hline 131 & $2.84437 e-6$ & $1.58373 e-4$ & 4.7309 \\
\hline 135 & $3.57238 \mathrm{e}-6$ & $1.93014 \mathrm{e}-4$ & 2.4942 \\
\hline 139 & $4.06086 e-6$ & $2.13093 e-4$ & 4.5897 \\
\hline 148 & $6.70991 \mathrm{e}-6$ & $3.30690 \mathrm{e}-4$ & 2.5036 \\
\hline 158 & $1.07905 e-5$ & $4.98138 \mathrm{e}-4$ & 2.1026 \\
\hline 173 & $1.77046 \mathrm{e}-5$ & $7.46460 \mathrm{e}-4$ & 2.0375 \\
\hline 178 & $2.00179 e-5$ & $8.20285 e-4$ & 3.2430 \\
\hline 188 & $2.84352 e-5$ & $1.10323 e-3$ & 11.8273 \\
\hline 192 & $3.17143 e-5$ & $1.20481 \mathrm{e}-3$ & 2.0691 \\
\hline 193 & $3.33284 e-5$ & $1.25957 \mathrm{e}-3$ & 2.5907 \\
\hline 198 & $4.10366 e-5$ & $1.51172 \mathrm{e}-3$ & 2.9568 \\
\hline 201 & $4.45584 e-5$ & $1.61696 \mathrm{e}-3$ & 2.4336 \\
\hline 202 & $4.50658 e-5$ & $1.62728 \mathrm{e}-3$ & 3.7049 \\
\hline 209 & $5.44802 e-5$ & $1.90133 e-3$ & 2.4039 \\
\hline 210 & $5.46214 \mathrm{e}-5$ & $1.89718 \mathrm{e}-3$ & 5.7344 \\
\hline
\end{tabular}

\begin{tabular}{|c|c|c|c|c|}
\hline 8 & 51 & $0.05 \%$ & 5 & 12 \\
\hline 22 & 254 & $0.15 \%$ & 18 & 69 \\
\hline 22 & 254 & $0.15 \%$ & 16 & 57 \\
\hline 14 & 131 & $0.09 \%$ & 11 & 24 \\
\hline 12 & 103 & $0.08 \%$ & 7 & 17 \\
\hline 16 & 161 & $0.11 \%$ & 12 & 31 \\
\hline 11 & 90 & $0.07 \%$ & 7 & 21 \\
\hline 17 & 177 & $0.11 \%$ & 12 & 29 \\
\hline 16 & 163 & $0.11 \%$ & 10 & 35 \\
\hline 6 & 31 & $0.04 \%$ & 3 & 5 \\
\hline 13 & 120 & $0.09 \%$ & 10 & 28 \\
\hline 13 & 120 & $0.09 \%$ & 9 & 28 \\
\hline 17 & 180 & $0.11 \%$ & 11 & 27 \\
\hline 2 & 2 & $0.01 \%$ & 1 & 1 \\
\hline 2 & 2 & $0.01 \%$ & 1 & 1 \\
\hline 2 & 2 & $0.01 \%$ & 1 & 1 \\
\hline 9 & 66 & $0.06 \%$ & 5 & 13 \\
\hline 20 & 228 & $0.13 \%$ & 9 & 31 \\
\hline 13 & 121 & $0.09 \%$ & 10 & 24 \\
\hline 11 & 93 & $0.07 \%$ & 8 & 17 \\
\hline 16 & 166 & $0.11 \%$ & 14 & 45 \\
\hline 5 & 22 & $0.03 \%$ & 4 & 6 \\
\hline 9 & 67 & $0.06 \%$ & 5 & 8 \\
\hline 6 & 32 & $0.04 \%$ & 3 & 5 \\
\hline 20 & 230 & $0.13 \%$ & 13 & 49 \\
\hline 7 & 43 & $0.05 \%$ & 5 & 7 \\
\hline 15 & 153 & $0.10 \%$ & 10 & 27 \\
\hline 4 & 14 & $0.03 \%$ & 2 & 3 \\
\hline 3 & 7 & $0.02 \%$ & 2 & 4 \\
\hline 5 & 23 & $0.03 \%$ & 3 & 5 \\
\hline 16 & 170 & $0.11 \%$ & 11 & 24 \\
\hline 9 & 54 & $0.06 \%$ & 6 & 9 \\
\hline 9 & 60 & $0.06 \%$ & 6 & 10 \\
\hline 8 & 50 & $0.05 \%$ & 4 & 8 \\
\hline 5 & 21 & $0.03 \%$ & 1 & 1 \\
\hline 5 & 21 & $0.03 \%$ & 1 & 1 \\
\hline 13 & 121 & $0.09 \%$ & 7 & 27 \\
\hline 9 & 68 & $0.06 \%$ & 6 & 14 \\
\hline 9 & 69 & $0.06 \%$ & 6 & 14 \\
\hline 36 & 304 & $0.24 \%$ & 27 & 73 \\
\hline 55 & 599 & $0.37 \%$ & 35 & 112 \\
\hline 21 & 148 & $0.14 \%$ & 14 & 41 \\
\hline 16 & 92 & $0.11 \%$ & 10 & 24 \\
\hline 45 & 499 & $0.30 \%$ & 32 & 90 \\
\hline 15 & 85 & $0.10 \%$ & 3 & 5 \\
\hline 13 & 65 & $0.09 \%$ & 2 & 5 \\
\hline 31 & 294 & $0.21 \%$ & 21 & 45 \\
\hline 13 & 67 & $0.09 \%$ & 2 & 3 \\
\hline 29 & 274 & $0.19 \%$ & 16 & 39 \\
\hline 40 & 450 & $0.27 \%$ & 28 & 80 \\
\hline 41 & 476 & $0.27 \%$ & 24 & 86 \\
\hline 17 & 124 & $0.11 \%$ & 6 & 16 \\
\hline 5 & 10 & $0.03 \%$ & 3 & 3 \\
\hline 37 & 423 & $0.25 \%$ & 22 & 69 \\
\hline 23 & 210 & $0.15 \%$ & 15 & 30 \\
\hline 18 & 144 & $0.12 \%$ & 7 & 20 \\
\hline 25 & 243 & $0.17 \%$ & 17 & 54 \\
\hline 13 & 83 & $0.09 \%$ & 7 & 17 \\
\hline 25 & 246 & $0.17 \%$ & 17 & 34 \\
\hline 8 & 33 & $0.05 \%$ & 5 & 10 \\
\hline
\end{tabular}


abnormal neocortex morphology decreased circulating dihydrotestosterone level absent femur

increased neurofibrosarcoma incidence

absent sacral vertebrae

decreased prepulse inhibition

prostate gland hyperplasia

intestinal adenocarcinoma

abnormal branchial arch artery morphology

decreased startle reflex

pancreatic ductal adenocarcinoma

small lung saccule

abnormal neural plate morphology

paraparesis

decreased pancreatic acinar cell number

muscle spasm

muscle twitch

ascites

abnormal prepulse inhibition

increased fibroma incidence

abnormal vertebral transverse process morphology

abnormal secondary ovarian follicle morphology

thin cerebral cortex

absent patella

abnormal liver development

abnormal rib development

distended pericardium

abnormal renal glomerulus basement membrane

morphology

abnormal pancreatic acinar cell morphology

abnormal ilium morphology

decreased aggression towards males

dermal cysts

enlarged prostate gland

decreased tongue size

pale liver

increased melanoma incidence

diarrhea

increased pancreatic islet number

short maxilla

abnormal pancreatic islet number

myoclonus

retinal ganglion cell degeneration

abnormal Rathke's pouch

abnormal bronchus epithelium morphology

abnormal rhombomere morphology

increased spindle cell carcinoma incidence

abnormal pulmonary respiratory rate

abnormal CNS synapse formation

squamous cell carcinoma

abnormal neuromere morphology

increased retinal ganglion cell number

increased mesothelioma incidence

increased cholangiocarcinoma incidence

decreased salivation

abnormal orientation of cochlear hair cell stereociliary bundles

decreased circulating estrogen level

ventricular hypoplasia

decreased renal tubule number

small limb buds

abnormal macrophage activation involved in immune

response

abnormal extraembryonic endoderm formation

abnormal pancreatic acinus morphology

abnormal pulmonary alveolar duct morphology

absent amacrine cells

abnormal susceptibility to hearing loss \begin{tabular}{|l|l|l|l|}
211 & $5.48779 \mathrm{e}-5$ & $1.89706 \mathrm{e}-3$ & 3.0010 \\
\hline
\end{tabular} \begin{tabular}{l|l|l|l}
227 & $7.55387 \mathrm{e}-5$ & $2.42722 \mathrm{e}-3$ & \\
\hline
\end{tabular} \begin{tabular}{|l|l|l|}
\hline 228 & $7.78070 \mathrm{e}-5$ & $2.48914 \mathrm{e}-3$ \\
\hline
\end{tabular} \begin{tabular}{l|l|l|}
229 & $7.93255 \mathrm{e}-5$ & $2.52664 \mathrm{e}-3$ \\
\hline
\end{tabular} \begin{tabular}{|c|c|c|}
234 & $8.30296 \mathrm{e}-5$ & $2.58811 \mathrm{e}-3$ \\
\hline
\end{tabular} $241 \quad 9.47000 \mathrm{e}-5 \mid \frac{2.86615 \mathrm{e}-3}{}$ \begin{tabular}{l|l|l|}
242 & $9.54443 \mathrm{e}-5$ & $2.87674 \mathrm{e}-3$ \\
\hline
\end{tabular} \begin{tabular}{|l|l|l|}
244 & $9.67834 \mathrm{e}-5$ & $2.89319 \mathrm{e}-3$ \\
\hline
\end{tabular} $249 \quad 1.09888 \mathrm{e}-4 \mid \frac{3.21896 \mathrm{e}-3}{}$ $250 \quad 1.10047 \mathrm{e}-4 \mid 3.21072 \mathrm{e}-3$ \begin{tabular}{|r|r|r|}
\hline 252 & $1.14220 \mathrm{e}-4$ & $3.30603 \mathrm{e}-3$ \\
\hline
\end{tabular} $252 \quad 1.14220 \mathrm{e}-4 \mid \frac{3.30603 \mathrm{e}-3}{}$ \begin{tabular}{r|r|r|}
255 & $1.17012 \mathrm{e}-4$ & $3.34700 \mathrm{e}-3$ \\
\hline
\end{tabular} \begin{tabular}{|l|l|l|}
\hline 256 & $1.18244 \mathrm{e}-4$ & $3.36902 \mathrm{e}-3$ \\
\hline
\end{tabular} \begin{tabular}{|r|r|r|}
\hline 260 & $1.30606 \mathrm{e}-4$ & $3.66400 \mathrm{e}-3$ \\
\hline
\end{tabular} \begin{tabular}{|l|l|l|}
\hline 264 & $1.36185 \mathrm{e}-4$ & $3.76263 \mathrm{e}-3$ \\
\hline
\end{tabular} \begin{tabular}{|l|l|l|}
264 & $1.36185 \mathrm{e}-4$ & $3.76263 \mathrm{e}-3$ \\
\hline
\end{tabular} \begin{tabular}{|l|l|l|}
271 & $1.56114 \mathrm{e}-4$ & $4.20183 \mathrm{e}-3$ \\
\hline
\end{tabular} \begin{tabular}{|l|l|l|}
272 & $1.58718 \mathrm{e}-4$ & $4.25620 \mathrm{e}-3$ \\
\hline
\end{tabular} \begin{tabular}{|l|l|l|}
\hline 273 & $1.61113 \mathrm{e}-4$ & $4.30460 \mathrm{e}-3$ \\
\hline
\end{tabular} \begin{tabular}{r|r|r|}
277 & $1.73448 \mathrm{e}-4$ & $4.56727 \mathrm{e}-3$ \\
\hline
\end{tabular} \begin{tabular}{|r|r|r|}
\hline 281 & $1.90339 \mathrm{e}-4$ & $4.94069 \mathrm{e}-3$ \\
\hline
\end{tabular} $291 \quad 2.31017 \mathrm{e}-4 \mid \frac{5.79052 \mathrm{e}-3}{}$ \begin{tabular}{|l|l|l|}
295 & $2.54205 \mathrm{e}-4$ & $6.28533 \mathrm{e}-3$ \\
\hline
\end{tabular} \begin{tabular}{|l|l|l|}
296 & $2.54422 \mathrm{e}-4$ & $6.26944 \mathrm{e}-3$ \\
\hline
\end{tabular} \begin{tabular}{|r|r|r|}
\hline 300 & $2.59553 \mathrm{e}-4$ & $6.31061 \mathrm{e}-3$ \\
\hline
\end{tabular} \begin{tabular}{|l|r|r|}
301 & $2.65088 \mathrm{e}-4$ & $6.42376 \mathrm{e}-3$ \\
\hline
\end{tabular} 23.6546 6.3685 4.8384 3.3116 2.4781 4.7309 2.4180 2.0625 2.3010 4.6281 4.6281 2.2921 3.0588 5.1145 2.0933 2.0933 2.5164 2.1365 4.4352 3.0950 2.9324 2.2351 3.1379 2.0763 2.2176 2.1741 \begin{tabular}{|l|l|l|}
\hline 304 & $2.81463 \mathrm{e}-4$ & $6.75325 \mathrm{e}-3$ \\
\hline
\end{tabular} 2.9568 \begin{tabular}{l|l|l|l|}
305 & $2.86314 \mathrm{e}-4$ & $6.84712 \mathrm{e}-3$ \\
\hline
\end{tabular} \begin{tabular}{|r|r|r|}
\hline 308 & $3.11046 \mathrm{e}-4$ & $7.36613 \mathrm{e}-3$ \\
\hline
\end{tabular} 2.4694 \begin{tabular}{|l|l|l|}
\hline 313 & $3.30789 \mathrm{e}-4$ & $7.70855 \mathrm{e}-3$ \\
\hline
\end{tabular} \begin{tabular}{|r|r|r|}
\hline 314 & $3.34650 \mathrm{e}-4$ & $7.77368 \mathrm{e}-3$ \\
\hline
\end{tabular} \begin{tabular}{|l|l|l|}
\hline 317 & $3.51985 \mathrm{e}-4$ & $8.09898 \mathrm{e}-3$ \\
\hline
\end{tabular} 3.0751 4.5056 3.2256 4.0168

\begin{tabular}{|l|l|l|l|}
\hline 322 & $3.78371 \mathrm{e}-4$ & $8.57092 \mathrm{e}-3$ & 3.0148 \\
\hline
\end{tabular}

\begin{tabular}{|l|l|l|l|}
\hline 323 & $3.79283 \mathrm{e}-4$ & $8.56499 \mathrm{e}-3$ & 2.0255 \\
\hline
\end{tabular}

\begin{tabular}{|r|r|r|r|r|}
\hline 325 & $3.91414 \mathrm{e}-4$ & $8.78454 \mathrm{e}-3$ & 4.4009 \\
\hline
\end{tabular}

\begin{tabular}{|r|r|r|r|r|}
\hline 327 & $4.03110 \mathrm{e}-4$ & $8.99170 \mathrm{e}-3$ & 2.4034 \\
\hline
\end{tabular}

\begin{tabular}{|r|r|r|}
328 & $4.06640 \mathrm{e}-4$ & $9.04277 \mathrm{e}-3$ \\
\hline
\end{tabular}

\begin{tabular}{|r|r|r|}
329 & $4.07177 \mathrm{e}-4$ & $9.02720 \mathrm{e}-3$ \\
\hline
\end{tabular}

3.9424

\begin{tabular}{r|r|r|}
334 & $4.17761 \mathrm{e}-4$ & $9.12320 \mathrm{e}-3$ \\
\hline
\end{tabular}

\begin{tabular}{|l|l|r|}
338 & $4.30879 \mathrm{e}-4$ & $9.29831 \mathrm{e}-3$ \\
\hline
\end{tabular}

2.1504

2.7294

\begin{tabular}{|c|c|c|}
339 & $4.37649 \mathrm{e}-4$ & $9.41655 \mathrm{e}-3$ \\
\hline
\end{tabular}

\begin{tabular}{|r|r|r|}
\hline 343 & $4.55211 \mathrm{e}-4$ & $9.68021 \mathrm{e}-3$ \\
\hline
\end{tabular}

\begin{tabular}{|l|l|l|}
\hline 344 & $4.57531 \mathrm{e}-4$ & $9.70126 \mathrm{e}-3$ \\
\hline
\end{tabular}

\begin{tabular}{|l|l|l|}
346 & $4.58474 \mathrm{e}-4$ & $9.66506 \mathrm{e}-3$ \\
\hline
\end{tabular}

\begin{tabular}{|l|l|l|}
\hline 347 & $4.72865 \mathrm{e}-4$ & $9.93970 \mathrm{e}-3$ \\
\hline
\end{tabular}

\begin{tabular}{|r|r|r|}
349 & $4.79120 \mathrm{e}-4$ & $1.00135 \mathrm{e}-2$ \\
\hline
\end{tabular}

\begin{tabular}{|l|l|l|}
351 & $4.88236 \mathrm{e}-4$ & $1.01458 \mathrm{e}-2$ \\
\hline
\end{tabular}

\begin{tabular}{|r|r|r|}
359 & $5.39685 \mathrm{e}-4$ & $1.09651 \mathrm{e}-2$ \\
\hline
\end{tabular}

\begin{tabular}{|l|c|c|}
369 & $5.87223 \mathrm{e}-4$ & $1.16076 \mathrm{e}-2$ \\
\hline
\end{tabular}

\begin{tabular}{r|r|r|}
372 & $6.13911 \mathrm{e}-4$ & $1.20373 \mathrm{e}-2$ \\
\hline
\end{tabular}

\begin{tabular}{|r|r|r|}
379 & $6.81452 \mathrm{e}-4$ & $1.31148 \mathrm{e}-2$ \\
\hline
\end{tabular}

2.3906

3.5840

2.4470

3.1193

2.3191

4.8701

2.2579

2.5923

2.2376

2.2745

3.7349

4.5995

\begin{tabular}{|r|r|r|}
\hline 379 & $6.81452 \mathrm{e}-4$ & $1.31148 \mathrm{e}-2$ \\
\hline
\end{tabular}

4.5995

\begin{tabular}{|l|l|l|}
\hline 384 & $7.06100 \mathrm{e}-4$ & $1.34122 \mathrm{e}-2$ \\
\hline
\end{tabular}

3.3792

\begin{tabular}{|l|l|l|}
392 & $7.64923 \mathrm{e}-4$ & $1.42330 \mathrm{e}-2$ \\
\hline
\end{tabular}

2.2814

\begin{tabular}{|l|l|l|}
393 & $7.85416 \mathrm{e}-4$ & $1.45772 \mathrm{e}-2$ \\
\hline 397 & $8.15635 \mathrm{e}-4$ & $1.49855 \mathrm{e}-2$ \\
\hline
\end{tabular}

2.5712

\begin{tabular}{l|l|l|r|}
397 & $8.15635 \mathrm{e}-4$ & $1.49855 \mathrm{e}-2$ & 2.4737 \\
\hline
\end{tabular}

\begin{tabular}{|l|l|l|r|r|}
\hline 399 & $8.25689 \mathrm{e}-4$ & $1.50942 \mathrm{e}-2$ & 3.0976 \\
\hline
\end{tabular}

\begin{tabular}{l|l|l|l|}
\hline 399 & $8.25689 \mathrm{e}-4$ & $1.50942 \mathrm{e}-2$ \\
\hline
\end{tabular}

3.0976

\begin{tabular}{l|l|l|l|}
403 & $8.44429 \mathrm{e}-4$ & $1.52835 \mathrm{e}-2$ & 3.9424 \\
\hline
\end{tabular}

\begin{tabular}{|l|l|l|l|}
\hline 406 & $8.80208 \mathrm{e}-4$ & $1.58134 \mathrm{e}-2$ & 2.3140 \\
\hline
\end{tabular}

\begin{tabular}{|l|l|l|l|}
\hline 408 & $8.88418 \mathrm{e}-4$ & $1.58826 \mathrm{e}-2$ & 2.2004 \\
\hline
\end{tabular}

\begin{tabular}{l|l|l|l|}
\hline 409 & $9.00428 \mathrm{e}-4$ & $1.60580 \mathrm{e}-2$ & 3.5482 \\
\hline
\end{tabular}

\begin{tabular}{|l|l|l|l|l|}
\hline 415 & $9.60969 \mathrm{e}-4$ & $1.68899 \mathrm{e}-2$ & 5.0688 \\
\hline
\end{tabular}

\begin{tabular}{l|l|l|}
\hline 420 & $9.97143 \mathrm{e}-4$ & $1.73170 \mathrm{e}-2$ \\
\hline
\end{tabular}

2.2892

\begin{tabular}{|c|c|c|c|c|}
\hline 17 & 134 & $0.11 \%$ & 9 & 25 \\
\hline 3 & 3 & $0.02 \%$ & 1 & 1 \\
\hline 7 & 26 & $0.05 \%$ & 4 & 4 \\
\hline 9 & 44 & $0.06 \%$ & 6 & 9 \\
\hline 14 & 100 & $0.09 \%$ & 3 & 9 \\
\hline 22 & 210 & $0.15 \%$ & 12 & 40 \\
\hline 9 & 45 & $0.06 \%$ & 7 & 10 \\
\hline 23 & 225 & $0.15 \%$ & 13 & 43 \\
\hline 32 & 367 & $0.21 \%$ & 21 & 52 \\
\hline 25 & 257 & $0.17 \%$ & 15 & 54 \\
\hline 9 & 46 & $0.06 \%$ & 6 & 12 \\
\hline 9 & 46 & $0.06 \%$ & 4 & 8 \\
\hline 25 & 258 & $0.17 \%$ & 16 & 35 \\
\hline 15 & 116 & $0.10 \%$ & 8 & 24 \\
\hline 8 & 37 & $0.05 \%$ & 2 & 6 \\
\hline 30 & 339 & $0.20 \%$ & 20 & 61 \\
\hline 30 & 339 & $0.20 \%$ & 20 & 62 \\
\hline 20 & 188 & $0.13 \%$ & 14 & 31 \\
\hline 28 & 310 & $0.19 \%$ & 17 & 63 \\
\hline 9 & 48 & $0.06 \%$ & 6 & 11 \\
\hline 14 & 107 & $0.09 \%$ & 5 & 7 \\
\hline 15 & 121 & $0.10 \%$ & 9 & 29 \\
\hline 24 & 254 & $0.16 \%$ & 15 & 65 \\
\hline 13 & 98 & $0.09 \%$ & 4 & 7 \\
\hline 28 & 319 & $0.19 \%$ & 21 & 55 \\
\hline 24 & 256 & $0.16 \%$ & 15 & 30 \\
\hline 25 & 272 & $0.17 \%$ & 20 & 59 \\
\hline 14 & 112 & $0.09 \%$ & 8 & 13 \\
\hline 19 & 182 & $0.13 \%$ & 11 & 38 \\
\hline 13 & 100 & $0.09 \%$ & 4 & 9 \\
\hline 8 & 42 & $0.05 \%$ & 6 & 13 \\
\hline 12 & 88 & $0.08 \%$ & 5 & 9 \\
\hline 9 & 53 & $0.06 \%$ & 7 & 16 \\
\hline 13 & 102 & $0.09 \%$ & 8 & 15 \\
\hline 28 & 327 & $0.19 \%$ & 23 & 75 \\
\hline 8 & 43 & $0.05 \%$ & 5 & 10 \\
\hline 19 & 187 & $0.13 \%$ & 13 & 41 \\
\hline 9 & 54 & $0.06 \%$ & 3 & 9 \\
\hline 24 & 264 & $0.16 \%$ & 16 & 42 \\
\hline 15 & 130 & $0.10 \%$ & 7 & 21 \\
\hline 19 & 188 & $0.13 \%$ & 11 & 31 \\
\hline 10 & 66 & $0.07 \%$ & 6 & 15 \\
\hline 18 & 174 & $0.12 \%$ & 11 & 25 \\
\hline 12 & 91 & $0.08 \%$ & 8 & 16 \\
\hline 20 & 204 & $0.13 \%$ & 13 & 25 \\
\hline 7 & 34 & $0.05 \%$ & 4 & 6 \\
\hline 21 & 220 & $0.14 \%$ & 16 & 44 \\
\hline 16 & 146 & $0.11 \%$ & 8 & 26 \\
\hline 21 & 222 & $0.14 \%$ & 10 & 45 \\
\hline 20 & 208 & $0.13 \%$ & 13 & 26 \\
\hline 9 & 57 & $0.06 \%$ & 4 & 10 \\
\hline 7 & 36 & $0.05 \%$ & 4 & 8 \\
\hline 7 & 36 & $0.05 \%$ & 5 & 6 \\
\hline 10 & 70 & $0.07 \%$ & 6 & 17 \\
\hline 19 & 197 & $0.13 \%$ & 11 & 36 \\
\hline 15 & 138 & $0.10 \%$ & 9 & 28 \\
\hline 16 & 153 & $0.11 \%$ & 9 & 29 \\
\hline 11 & 84 & $0.07 \%$ & 4 & 6 \\
\hline 11 & 84 & $0.07 \%$ & 7 & 12 \\
\hline 8 & 48 & $0.05 \%$ & 4 & 7 \\
\hline 18 & 184 & $0.12 \%$ & 10 & 35 \\
\hline 20 & 215 & $0.13 \%$ & 12 & 44 \\
\hline 9 & 60 & $0.06 \%$ & 4 & 14 \\
\hline 6 & 28 & $0.04 \%$ & 1 & 4 \\
\hline 18 & 186 & $0.12 \%$ & 13 & 34 \\
\hline
\end{tabular}


abnormal prostate gland size abnormal heart shape

abnormal pancreas development

increased hepatocyte apoptosis

pancreatic intraepithelial neoplasia

abnormal primary somatosensory cortex morphology

increased vertebrae number

abnormal conotruncus morphology

failure of palatal shelf elevation

cleft chin

abnormal circulating potassium level

increased aggression

abnormal alveolocapillary membrane morphology

abnormal serous gland morphology

mammary gland hyperplasia

arteritis

enlarged lung

short sternum

increased grooming behavior

increased glioblastoma incidence

patent ductus arteriosus

abnormal cortical ventricular zone morphology

absent ulna

abnormal cricoid cartilage morphology

increased keratinocyte proliferation

enlarged hair follicles

progressive muscle weakness

vasculitis

muscular ventricular septal defect

kidney medulla hypoplasia

abnormal hippocampal fimbria

impaired branching involved in terminal bronchiole

morphogenesis

small ischium

absent awl hair

delayed male fertility

absent auchene hairs

L5 dorsal root ganglion hypertrophy

enlarged prostate gland anterior lobe

abnormal autophagy

increased susceptibility to noise-induced hearing loss

uterus adenomyosis

increased neurofibroma incidence

increased pulmonary respiratory rate

abnormal intestinal goblet cells

abnormal sternum ossification

abnormal oviduct morphology

increased prostate gland tumor incidence

absent ureter

abnormal orientation of outer hair cell stereociliary

bundles

reduced linear vestibular evoked potential

pancreas cysts

increased cochlear nerve compound action potential

abnormal memory B cell morphology

small pubis

social withdrawal

abnormal visual cortex morphology

absent retinal inner plexiform layer

skeletal muscle fibrosis

abnormal auditory cortex morphology

absent ilium

abnormal extraocular muscle morphology

necrospermia

hyperpigmentation

decreased synaptic depression

abnormal second branchial arch morphology

prostate gland adenocarcinoma \begin{tabular}{|l|l|l|}
422 & $1.00472 \mathrm{e}-3$ & $1.73660 \mathrm{e}-2$ \\
\hline
\end{tabular} \begin{tabular}{l|l|l|l}
426 & $1.05430 \mathrm{e}-3$ & $1.80518 \mathrm{e}-2$ & \\
\hline & &
\end{tabular}

2.4261 \begin{tabular}{l|l|l|l|}
\hline 429 & $1.06177 \mathrm{e}-3$ & $1.80525 \mathrm{e}-2$ \\
\hline
\end{tabular} \begin{tabular}{|l|l|l|}
\hline 432 & $1.08158 \mathrm{e}-3$ & $1.82617 \mathrm{e}-2$ \\
\hline 437 & $1.14561 \mathrm{e}-3$ & $1.91214 \mathrm{e}-2$ \\
\hline
\end{tabular} \begin{tabular}{|l|l|l|}
\hline 437 & $1.14561 \mathrm{e}-3$ & $1.91214 \mathrm{e}-2$ \\
\hline
\end{tabular} \begin{tabular}{|l|r|r|}
\hline 438 & $1.17316 \mathrm{e}-3$ & $1.95366 \mathrm{e}-2$ \\
\hline
\end{tabular} \begin{tabular}{l|l|l|l|}
\hline 439 & $1.17358 \mathrm{e}-3$ & $1.94990 \mathrm{e}-2$ \\
\hline
\end{tabular} \begin{tabular}{|l|l|l|}
\hline 441 & $1.18148 \mathrm{e}-3$ & $1.95414 \mathrm{e}-2$ \\
\hline
\end{tabular} \begin{tabular}{l|r|r|}
443 & $1.19726 \mathrm{e}-3$ & $1.97128 \mathrm{e}-2$ \\
\hline
\end{tabular} \begin{tabular}{l|c|c|}
\hline 447 & $1.20199 \mathrm{e}-3$ & $1.96136 \mathrm{e}-2$ \\
\hline
\end{tabular} \begin{tabular}{l|r|r|}
448 & $1.21550 \mathrm{e}-3$ & $1.97898 \mathrm{e}-2$
\end{tabular} \begin{tabular}{|c|c|c|}
449 & $1.22190 \mathrm{e}-3$ & $1.98498 \mathrm{e}-2$ \\
\hline
\end{tabular} \begin{tabular}{l|l|l|}
450 & $1.22691 \mathrm{e}-3$ & $1.98869 \mathrm{e}-2$ \\
\hline
\end{tabular} \begin{tabular}{|l|r|r|}
\hline 450 & $1.22691 \mathrm{e}-3$ & $1.98869 \mathrm{e}-2$ \\
\hline
\end{tabular} \begin{tabular}{|r|r|r|}
\hline 454 & $1.27913 \mathrm{e}-3$ & $2.05506 \mathrm{e}-2$ \\
\hline
\end{tabular} \begin{tabular}{|l|l|l|}
455 & $1.31314 \mathrm{e}-3$ & $2.10507 \mathrm{e}-2$ \\
\hline
\end{tabular} \begin{tabular}{l|r|r|}
460 & $1.37642 \mathrm{e}-3$ & $2.18253 \mathrm{e}-2$ \\
\hline
\end{tabular} \begin{tabular}{l|l|l|}
\hline 461 & $1.38266 \mathrm{e}-3$ & $2.18766 \mathrm{e}-2$ \\
\hline
\end{tabular} \begin{tabular}{|l|l|l|}
463 & $1.39289 \mathrm{e}-3$ & $2.19434 \mathrm{e}-2$ \\
\hline
\end{tabular} \begin{tabular}{l|l|l|l|}
\hline 464 & $1.40884 \mathrm{e}-3$ & $2.21467 \mathrm{e}-2$ \\
\hline
\end{tabular} \begin{tabular}{|l|l|l|}
\hline 466 & $1.44206 \mathrm{e}-3$ & $2.25716 \mathrm{e}-2$ \\
\hline
\end{tabular} \begin{tabular}{|l|r|r|}
\hline 466 & $1.44206 \mathrm{e}-3$ & $2.25716 \mathrm{e}-2$ \\
\hline
\end{tabular} \begin{tabular}{|r|r|r|}
\hline 471 & $1.49474 \mathrm{e}-3$ & $2.31479 \mathrm{e}-2$ \\
\hline
\end{tabular} \begin{tabular}{|l|r|r|}
\hline 472 & $1.49573 \mathrm{e}-3$ & $2.31140 \mathrm{e}-2$ \\
\hline
\end{tabular} \begin{tabular}{l|l|l|}
472 & $1.49573 \mathrm{e}-3$ & $2.31140 \mathrm{e}-2$ \\
\hline
\end{tabular} \begin{tabular}{|r|r|r|}
\hline 479 & $1.55454 \mathrm{e}-3$ & $2.36718 \mathrm{e}-2$ \\
\hline
\end{tabular} \begin{tabular}{|l|l|l|}
\hline 485 & $1.60744 \mathrm{e}-3$ & $2.41746 \mathrm{e}-2$ \\
\hline
\end{tabular} \begin{tabular}{|l|l|l|}
\hline 485 & $1.60744 \mathrm{e}-3$ & $2.41746 \mathrm{e}-2$ \\
\hline
\end{tabular} \begin{tabular}{|l|l|l|}
\hline 488 & $1.62902 \mathrm{e}-3$ & $2.43485 \mathrm{e}-2$ \\
\hline
\end{tabular} \begin{tabular}{r|r|r|}
\hline 490 & $1.64552 \mathrm{e}-3$ & $2.44948 \mathrm{e}-2$ \\
\hline
\end{tabular} \begin{tabular}{l|l|l|}
491 & $1.65047 \mathrm{e}-3$ & $2.45184 \mathrm{e}-2$ \\
\hline
\end{tabular} \begin{tabular}{|l|l|l|}
\hline 491 & $1.65047 \mathrm{e}-3$ & $2.45184 \mathrm{e}-2$ \\
\hline
\end{tabular} \begin{tabular}{|c|c|c|}
\hline 497 & $1.67820 \mathrm{e}-3$ & $2.46294 \mathrm{e}-2$ \\
\hline
\end{tabular} \begin{tabular}{|c|c|c|}
497 & $1.67820 \mathrm{e}-3$ & $2.46294 \mathrm{e}-2$ \\
\hline
\end{tabular} \begin{tabular}{|l|l|l|}
497 & $1.67820 \mathrm{e}-3$ & $2.46294 \mathrm{e}-2$ \\
\hline
\end{tabular} \begin{tabular}{|l|l|l|}
500 & $1.68497 \mathrm{e}-3$ & $2.45803 \mathrm{e}-2$ \\
\hline
\end{tabular} \begin{tabular}{r|r|r|}
504 & $1.78707 \mathrm{e}-3$ & $2.58628 \mathrm{e}-2$ \\
\hline
\end{tabular} \begin{tabular}{|l|r|r|}
504 & $1.78707 \mathrm{e}-3$ & $2.58628 \mathrm{e}-2$ \\
\hline
\end{tabular} \begin{tabular}{|l|l|l|}
512 & $1.91772 \mathrm{e}-3$ & $2.73200 \mathrm{e}-2$ \\
\hline
\end{tabular} \begin{tabular}{|l|l|l|}
515 & $1.99925 \mathrm{e}-3$ & $2.83155 \mathrm{e}-2$ \\
\hline
\end{tabular} \begin{tabular}{|l|l|l|}
517 & $2.03250 \mathrm{e}-3$ & $2.86752 \mathrm{e}-2$ \\
\hline
\end{tabular} \begin{tabular}{l|l|l|}
517 & $2.03250 \mathrm{e}-3$ & $2.86752 \mathrm{e}-2$ \\
\hline
\end{tabular} \begin{tabular}{l|l|l|}
519 & $2.03907 \mathrm{e}-3$ & $2.86570 \mathrm{e}-2$ \\
\hline
\end{tabular} \begin{tabular}{l|l|l|}
520 & $2.06734 \mathrm{e}-3$ & $2.89984 \mathrm{e}-2$ \\
\hline
\end{tabular} \begin{tabular}{l|l|r|}
520 & $2.06734 \mathrm{e}-3$ & $2.89984 \mathrm{e}-2$ \\
\hline
\end{tabular} $523 \quad 2.09018 \mathrm{e}-3 \quad 2.91507 \mathrm{e}-2$ \begin{tabular}{|l|l|l|}
523 & $2.09018 \mathrm{e}-3$ & $2.91507 \mathrm{e}-2$ \\
\hline 530 & $2.19329 \mathrm{e}-3$ & $3.01846 \mathrm{e}-2$ \\
\hline
\end{tabular} \begin{tabular}{l|l|l|}
530 & $2.19329 \mathrm{e}-3$ & $3.01846 \mathrm{e}-2$ \\
\hline
\end{tabular} 2.4987 2.0060 3.4337 2.8104 2.5672 2.1049 2.2528 7.8849 2.4640 2.3111 5.9137 5.9137 2.7829 4.1396 2.1712 2.0784 2.7559 4.7309 3.3264 3.3264 3.0720 2.3363 2.3363 5.6320 2.8593 2.8593 2.5841 2.7034 3.0326 3.0326 7.2783 7.2783 7.2783 4.5783 23.6546 23.6546 2.7979 3.1775 3.8507 3.8507 2.5206 2.0111 2.0111 2.7681 2.7681 2.5001 \begin{tabular}{l|l|l|l|}
530 & $2.19329 \mathrm{e}-3$ & $3.01846 \mathrm{e}-2$ & 2.5001
\end{tabular} \begin{tabular}{l|l|l|}
532 & $2.19798 \mathrm{e}-3$ & $3.01355 \mathrm{e}-2$ \\
\hline
\end{tabular} 2.9203 \begin{tabular}{l|l|l|}
537 & $2.27502 \mathrm{e}-3$ & $3.09014 \mathrm{e}-2$ \\
\hline
\end{tabular} \begin{tabular}{l|l|l|}
540 & $2.32522 \mathrm{e}-3$ & $3.14077 \mathrm{e}-2$ \\
\hline
\end{tabular} \begin{tabular}{|l|l|l|}
541 & $2.32986 \mathrm{e}-3$ & $3.14121 \mathrm{e}-2$ \\
\hline
\end{tabular} \begin{tabular}{|l|r|r|}
541 & $2.32986 \mathrm{e}-3$ & $3.14121 \mathrm{e}-2$ \\
\hline
\end{tabular} \begin{tabular}{l|l|l|}
543 & $2.35793 \mathrm{e}-3$ & $3.16736 \mathrm{e}-2$ \\
\hline
\end{tabular} \begin{tabular}{|c|c|c|}
544 & $2.35819 \mathrm{e}-3$ & $3.16189 \mathrm{e}-2$ \\
\hline
\end{tabular} \begin{tabular}{|l|l|l|}
544 & $2.35819 \mathrm{e}-3$ & $3.16189 \mathrm{e}-2$ \\
\hline
\end{tabular} \begin{tabular}{|l|r|r|}
547 & $2.38561 \mathrm{e}-3$ & $3.18110 \mathrm{e}-2$ \\
\hline
\end{tabular} \begin{tabular}{|l|l|l|}
548 & $2.39588 \mathrm{e}-3$ & $3.18897 \mathrm{e}-2$ \\
\hline
\end{tabular} \begin{tabular}{|l|l|l|}
548 & $2.39588 \mathrm{e}-3$ & $3.18897 \mathrm{e}-2$ \\
\hline
\end{tabular} \begin{tabular}{|l|l|l|}
548 & $2.39588 \mathrm{e}-3$ & $3.18897 \mathrm{e}-2$ \\
\hline
\end{tabular} \begin{tabular}{|l|l|l|}
548 & $2.39588 \mathrm{e}-3$ & $3.18897 \mathrm{e}-2$ \\
\hline
\end{tabular} \begin{tabular}{|l|l|l|}
552 & $2.44524 \mathrm{e}-3$ & $3.23109 \mathrm{e}-2$ \\
\hline
\end{tabular} \begin{tabular}{|l|l|l|}
555 & $2.45752 \mathrm{e}-3$ & $3.22976 \mathrm{e}-2$ \\
\hline
\end{tabular} \begin{tabular}{|l|r|r|}
561 & $2.63810 \mathrm{e}-3$ & $3.43001 \mathrm{e}-2$ \\
\hline
\end{tabular} 2.7390 10.1377 3.7632 3.7632 3.3792 4.3008 4.3008 2.3825 5.1423 5.1423 5.1423 5.1423 2.5805 3.0854 2.1504 3.3199

\begin{tabular}{|c|c|c|c|c|}
\hline 16 & 156 & $0.11 \%$ & 13 & 37 \\
\hline 15 & 142 & $0.10 \%$ & 12 & 29 \\
\hline 21 & 234 & $0.14 \%$ & 12 & 32 \\
\hline 24 & 283 & $0.16 \%$ & 18 & 56 \\
\hline 9 & 62 & $0.06 \%$ & 6 & 11 \\
\hline 12 & 101 & $0.08 \%$ & 9 & 18 \\
\hline 14 & 129 & $0.09 \%$ & 3 & 13 \\
\hline 21 & 236 & $0.14 \%$ & 11 & 33 \\
\hline 18 & 189 & $0.12 \%$ & 10 & 29 \\
\hline 4 & 12 & $0.03 \%$ & 2 & 3 \\
\hline 15 & 144 & $0.10 \%$ & 9 & 29 \\
\hline 17 & 174 & $0.11 \%$ & 15 & 36 \\
\hline 5 & 20 & $0.03 \%$ & 2 & 3 \\
\hline 5 & 20 & $0.03 \%$ & 3 & 3 \\
\hline 12 & 102 & $0.08 \%$ & 8 & 18 \\
\hline 7 & 40 & $0.05 \%$ & 5 & 13 \\
\hline 19 & 207 & $0.13 \%$ & 11 & 48 \\
\hline 21 & 239 & $0.14 \%$ & 13 & 39 \\
\hline 12 & 103 & $0.08 \%$ & 7 & 16 \\
\hline 6 & 30 & $0.04 \%$ & 4 & 7 \\
\hline 9 & 64 & $0.06 \%$ & 6 & 16 \\
\hline 9 & 64 & $0.06 \%$ & 5 & 10 \\
\hline 10 & 77 & $0.07 \%$ & 3 & 8 \\
\hline 16 & 162 & $0.11 \%$ & 9 & 17 \\
\hline 16 & 162 & $0.11 \%$ & 9 & 31 \\
\hline 5 & 21 & $0.03 \%$ & 2 & 4 \\
\hline 11 & 91 & $0.07 \%$ & 9 & 29 \\
\hline 11 & 91 & $0.07 \%$ & 10 & 25 \\
\hline 13 & 119 & $0.09 \%$ & 7 & 18 \\
\hline 12 & 105 & $0.08 \%$ & 5 & 8 \\
\hline 10 & 78 & $0.07 \%$ & 6 & 10 \\
\hline 10 & 78 & $0.07 \%$ & 5 & 11 \\
\hline 4 & 13 & $0.03 \%$ & 1 & 2 \\
\hline 4 & 13 & $0.03 \%$ & 1 & 1 \\
\hline 4 & 13 & $0.03 \%$ & 3 & 6 \\
\hline 6 & 31 & $0.04 \%$ & 2 & 5 \\
\hline 2 & 2 & $0.01 \%$ & 1 & 1 \\
\hline 2 & 2 & $0.01 \%$ & 2 & 2 \\
\hline 11 & 93 & $0.07 \%$ & 6 & 22 \\
\hline 9 & 67 & $0.06 \%$ & 6 & 13 \\
\hline 7 & 43 & $0.05 \%$ & 4 & 6 \\
\hline 7 & 43 & $0.05 \%$ & 5 & 8 \\
\hline 13 & 122 & $0.09 \%$ & 10 & 25 \\
\hline 21 & 247 & $0.14 \%$ & 12 & 43 \\
\hline 21 & 247 & $0.14 \%$ & 13 & 39 \\
\hline 11 & 94 & $0.07 \%$ & 8 & 18 \\
\hline 11 & 94 & $0.07 \%$ & 6 & 26 \\
\hline 13 & 123 & $0.09 \%$ & 7 & 18 \\
\hline 13 & 123 & $0.09 \%$ & 8 & 24 \\
\hline 10 & 81 & $0.07 \%$ & 5 & 13 \\
\hline 11 & 95 & $0.07 \%$ & 7 & 22 \\
\hline 3 & 7 & $0.02 \%$ & 2 & 2 \\
\hline 7 & 44 & $0.05 \%$ & 5 & 14 \\
\hline 7 & 44 & $0.05 \%$ & 4 & 7 \\
\hline 8 & 56 & $0.05 \%$ & 7 & 8 \\
\hline 6 & 33 & $0.04 \%$ & 3 & 7 \\
\hline 6 & 33 & $0.04 \%$ & 1 & 4 \\
\hline 14 & 139 & $0.09 \%$ & 10 & 26 \\
\hline 5 & 23 & $0.03 \%$ & 2 & 4 \\
\hline 5 & 23 & $0.03 \%$ & 2 & 2 \\
\hline 5 & 23 & $0.03 \%$ & 4 & 5 \\
\hline 5 & 23 & $0.03 \%$ & 2 & 5 \\
\hline 12 & 110 & $0.08 \%$ & 8 & 27 \\
\hline 9 & 69 & $0.06 \%$ & 5 & 10 \\
\hline 17 & 187 & $0.11 \%$ & 13 & 37 \\
\hline 8 & 57 & $0.05 \%$ & 5 & 15 \\
\hline
\end{tabular}


|abnormal pancreatic beta cell differentiation abnormal nest building behavior

abnormal anterior visceral endoderm morphology

abnormal accessory nerve morphology

bronchiolar epithelial hyperplasia

abnormal vestibulocochlear nerve morphology

small vestibular ganglion

abnormal pancreatic alpha cell morphology

abnormal barrel cortex morphology

proctitis

increased grip strength

increased stomach tumor incidence

decreased circulating follicle stimulating hormone level

abnormal amacrine cell morphology

decreased follicle stimulating hormone level

abnormal cochlear nerve morphology

tachypnea

abnormal penis morphology

pale placenta

fusion of glossopharyngeal and vagus nerve

abnormal parietal lobe morphology

decreased circulating sodium level

abnormal interventricular septum muscular part

morphology

decreased circulating antidiuretic hormone level

impaired synaptic plasticity

abnormal sesamoid bone of gastrocnemius

morphology

abnormal endplate potential

absent ureteric bud

abnormal vagina opening

failure of atrioventricular cushion closure

constricted vagina opening

vascular inflammation

darkened coat color

decreased pulmonary ventilation

abnormal branching involved in terminal bronchiole

morphogenesis

abnormal vascular regression

abnormal fetal ductus arteriosus morphology

abnormal ureter development

abnormal occipital lobe morphology

absent branchial arches

increased fibrosarcoma incidence

abnormal hair follicle outer root sheath morphology

hyperresponsive to tactile stimuli

abnormal conotruncus septation

Conn syndrome

middle ear disease

primary Escherichia coli infectious disease

commensal Escherichia coli infectious disease

primary Enterobacteriaceae infectious disease

myopia

otitis media

refractive error

Hashimoto Disease

cervical adenocarcinoma

glandular cystitis

mucinous adenocarcinoma

Hirschsprung's disease

actinic keratosis

Burkitt's lymphoma

endemic goiter

background diabetic retinopathy

chronic cystitis

Charcot-Marie-Tooth disease

megacolon \begin{tabular}{|l|l|l|}
565 & $2.65961 \mathrm{e}-3$ & $3.43349 \mathrm{e}-2$ \\
\hline
\end{tabular} \begin{tabular}{l|l|l|}
567 & $2.68441 \mathrm{e}-3$ & $3.45328 \mathrm{e}-2$ \\
\hline
\end{tabular} \begin{tabular}{l|l|l|}
567 & $2.68441 \mathrm{e}-3$ & $3.45328 \mathrm{e}-2$ \\
\hline
\end{tabular} \begin{tabular}{|l|l|l|}
571 & $2.76219 \mathrm{e}-3$ & $3.52845 \mathrm{e}-2$ \\
\hline
\end{tabular} \begin{tabular}{|l|l|l|}
\hline 571 & $2.76219 \mathrm{e}-3$ & $3.52845 \mathrm{e}-2$ \\
\hline
\end{tabular} \begin{tabular}{|l|l|l|}
574 & $2.84288 \mathrm{e}-3$ & $3.61254 \mathrm{e}-2$ \\
\hline
\end{tabular} \begin{tabular}{|l|l|l|}
577 & $2.88273 e-3$ & $3.64413 e-2$ \\
\hline
\end{tabular} \begin{tabular}{l|l|l|}
578 & $2.89454 \mathrm{e}-3$ & $3.65272 \mathrm{e}-2$ \\
\hline
\end{tabular} \begin{tabular}{l|l|l|}
579 & $2.91029 \mathrm{e}-3$ & $3.66627 \mathrm{e}-2$ \\
\hline
\end{tabular} \begin{tabular}{|l|l|l|}
580 & $2.92191 \mathrm{e}-3$ & $3.67456 \mathrm{e}-2$ \\
\hline
\end{tabular} \begin{tabular}{|l|l|l|}
\hline 580 & $2.92191 \mathrm{e}-3$ & $3.67456 \mathrm{e}-2$ \\
\hline
\end{tabular} \begin{tabular}{l|l|l|}
589 & $3.15121 \mathrm{e}-3$ & $3.90237 \mathrm{e}-2$ \\
\hline
\end{tabular} \begin{tabular}{|l|l|l|}
593 & $3.21411 \mathrm{e}-3$ & $3.95340 \mathrm{e}-2$ \\
\hline
\end{tabular} \begin{tabular}{|l|l|l|}
595 & $3.27208 \mathrm{e}-3$ & $4.01119 \mathrm{e}-2$ \\
\hline
\end{tabular} \begin{tabular}{|l|l|l|}
596 & $3.28220 \mathrm{e}-3$ & $4.01684 \mathrm{e}-2$ \\
\hline
\end{tabular} \begin{tabular}{|l|l|l|}
598 & $3.29217 \mathrm{e}-3$ & $4.01557 \mathrm{e}-2$ \\
\hline
\end{tabular} \begin{tabular}{|l|l|l|}
599 & $3.29694 \mathrm{e}-3$ & $4.01467 \mathrm{e}-2$ \\
\hline
\end{tabular} \begin{tabular}{|l|l|l|}
\hline 600 & $3.29974 \mathrm{e}-3$ & $4.01138 \mathrm{e}-2$ \\
\hline
\end{tabular} \begin{tabular}{|c|c|c|}
\hline 604 & $3.40788 \mathrm{e}-3$ & $4.11541 \mathrm{e}-2$ \\
\hline
\end{tabular} \begin{tabular}{|c|c|c|}
604 & $3.40788 \mathrm{e}-3$ & $4.11541 \mathrm{e}-2$ \\
\hline
\end{tabular} \begin{tabular}{|l|l|l|}
608 & $3.46087 \mathrm{e}-3$ & $4.15191 \mathrm{e}-2$ \\
\hline
\end{tabular} \begin{tabular}{|l|l|l|}
\hline 611 & $3.52648 \mathrm{e}-3$ & $4.20984 \mathrm{e}-2$ \\
\hline
\end{tabular}

\begin{tabular}{l|l|l|}
613 & $3.56216 \mathrm{e}-3$ & $4.23856 \mathrm{e}-2$ \\
\hline
\end{tabular}

\begin{tabular}{|l|l|l|}
\hline 616 & $3.60345 e-3$ & $4.26682 \mathrm{e}-2$ \\
\hline
\end{tabular}

\begin{tabular}{l|l|l|}
618 & $3.65002 \mathrm{e}-3$ & $4.30797 \mathrm{e}-2$ \\
\hline
\end{tabular}

\begin{tabular}{l|l|l|}
619 & $3.66013 e-3$ & $4.31292 e-2$
\end{tabular}

\begin{tabular}{l|l|l|l|}
624 & $3.79536 \mathrm{e}-3$ & $4.43644 \mathrm{e}-2$ \\
\hline
\end{tabular}

\begin{tabular}{|l|l|l|}
625 & $3.80456 \mathrm{e}-3$ & $4.44007 \mathrm{e}-2$ \\
\hline
\end{tabular}

\begin{tabular}{|l|l|l|}
\hline 627 & $3.84630 \mathrm{e}-3$ & $4.47446 \mathrm{e}-2$ \\
\hline
\end{tabular}

\begin{tabular}{|l|l|l|}
\hline 629 & $3.86602 \mathrm{e}-3$ & $4.48311 \mathrm{e}-2$ \\
\hline
\end{tabular}

\begin{tabular}{|l|l|l|}
\hline 629 & $3.86602 \mathrm{e}-3$ & $4.48311 \mathrm{e}-2$ \\
\hline
\end{tabular}

\begin{tabular}{|l|l|l|}
\hline 634 & $3.97135 e-3$ & $4.56893 e-2$ \\
\hline
\end{tabular}

\begin{tabular}{|l|l|l|}
\hline 634 & $3.97135 \mathrm{e}-3$ & $4.56893 \mathrm{e}-2$ \\
\hline
\end{tabular}

\begin{tabular}{l|l|l|}
639 & $4.04930 \mathrm{e}-3$ & $4.62216 \mathrm{e}-2$ \\
\hline
\end{tabular}

\begin{tabular}{|l|l|l|}
\hline 639 & $4.04930 \mathrm{e}-3$ & $4.62216 \mathrm{e}-2$ \\
\hline
\end{tabular}

\begin{tabular}{l|l|l|}
\hline 643 & $4.08169 \mathrm{e}-3$ & $4.63015 \mathrm{e}-2$ \\
\hline
\end{tabular}

\begin{tabular}{|l|l|l|}
\hline 649 & $4.22140 \mathrm{e}-3$ & $4.74436 \mathrm{e}-2$ \\
\hline
\end{tabular}

\begin{tabular}{|l|l|l|}
652 & $4.26493 e-3$ & $4.77123 e-2$ \\
\hline
\end{tabular}

\begin{tabular}{l|l|l|}
653 & $4.28646 e-3$ & $4.78797 e-2$ \\
\hline
\end{tabular}

\begin{tabular}{|l|l|l|}
656 & $4.34676 \mathrm{e}-3$ & $4.83312 \mathrm{e}-2$ \\
\hline
\end{tabular}

\begin{tabular}{l|l|l|l|}
656 & $4.34676 \mathrm{e}-3$ & $4.83312 \mathrm{e}-2$ \\
\hline
\end{tabular}

\begin{tabular}{|l|l|l|}
660 & $4.39232 \mathrm{e}-3$ & $4.85418 \mathrm{e}-2$ \\
\hline
\end{tabular}

\begin{tabular}{|l|l|l|}
664 & $4.49071 \mathrm{e}-3$ & $4.93302 \mathrm{e}-2$ \\
\hline
\end{tabular}

\begin{tabular}{l|l|l|}
664 & $4.49071 \mathrm{e}-3$ & $4.93302 \mathrm{e}-2$
\end{tabular}

\begin{tabular}{l|l|l|}
44 & $5.07396 \mathrm{e}-6$ & $2.57619 \mathrm{e}-4$ \\
\hline
\end{tabular}

\begin{tabular}{l|l|l|}
56 & $9.52958 \mathrm{e}-6$ & $3.80162 \mathrm{e}-4$ \\
\hline
\end{tabular}

\begin{tabular}{l|l|l|l|}
68 & $1.94168 \mathrm{e}-5$ & $6.37899 \mathrm{e}-4$ \\
\hline
\end{tabular}

\begin{tabular}{l|l|l|l}
68 & $1.94168 e-5$ & $6.37899 e-4$
\end{tabular}

\begin{tabular}{l|l|l|}
79 & $4.52652 \mathrm{e}-5$ & $1.28003 \mathrm{e}-3$ \\
\hline
\end{tabular}

\begin{tabular}{l|l|l|}
83 & $5.33988 \mathrm{e}-5$ & $1.43726 \mathrm{e}-3$
\end{tabular}

\begin{tabular}{l|l|l|}
89 & $8.40485 e-5$ & $2.10971 e-3$ \\
\hline
\end{tabular}

\begin{tabular}{r|r|r|}
\hline 94 & $1.88447 \mathrm{e}-4$ & $4.47864 \mathrm{e}-3$ \\
\hline
\end{tabular}

\begin{tabular}{l|l|l|}
110 & $5.67576 \mathrm{e}-4$ & $1.15270 \mathrm{e}-2$ \\
\hline
\end{tabular}

\begin{tabular}{|l|l|l|}
\hline 127 & $9.12613 \mathrm{e}-4$ & $1.60534 \mathrm{e}-2$ \\
\hline
\end{tabular}

\begin{tabular}{l|l|l|}
\hline 134 & $1.11481 \mathrm{e}-3$ & $1.85857 \mathrm{e}-2$ \\
\hline
\end{tabular}

\begin{tabular}{|l|l|l|}
\hline 135 & $1.14561 \mathrm{e}-3$ & $1.89577 \mathrm{e}-2$ \\
\hline
\end{tabular}

\begin{tabular}{l|l|l|}
137 & $1.39556 \mathrm{e}-3$ & $2.27567 \mathrm{e}-2$ \\
\hline
\end{tabular}

\begin{tabular}{|l|l|l|}
\hline 139 & $1.44206 \mathrm{e}-3$ & $2.31766 \mathrm{e}-2$ \\
\hline
\end{tabular}

\begin{tabular}{l|l|l|}
\hline 140 & $1.56432 \mathrm{e}-3$ & $2.49621 \mathrm{e}-2$ \\
\hline
\end{tabular}

\begin{tabular}{l|l|l|}
145 & $1.78707 \mathrm{e}-3$ & $2.75332 \mathrm{e}-2$ \\
\hline
\end{tabular}

\begin{tabular}{l|l|l|}
\hline 145 & $1.78707 \mathrm{e}-3$ & $2.75332 \mathrm{e}-2$ \\
\hline
\end{tabular}

\begin{tabular}{|l|r|r|}
\hline 147 & $1.81908 \mathrm{e}-3$ & $2.76450 \mathrm{e}-2$ \\
\hline
\end{tabular}

\begin{tabular}{|l|l|l|}
\hline 149 & $1.87129 \mathrm{e}-3$ & $2.80567 \mathrm{e}-2$ \\
\hline
\end{tabular}

\begin{tabular}{l|l|l|}
150 & $1.89389 \mathrm{e}-3$ & $2.82064 \mathrm{e}-2$ \\
\hline
\end{tabular}
3.6796

2.6825

2.6825

4.1743

4.1743

2.5344

2.8160

2.0337

2.6551

4.9280

4.9280

2.6283

2.2316

2.1627

2.1054

3.2074

2.9568

2.2998

2.6020

2.6020

2.1504

4.7309

2.3655

8.8705

2.0836

3.1539

2.4470

2.3474

2.0728

3.4496

3.4496

2.5510

2.5510

2.6880

2.6880

2.1144

2.2376

2.0517

3.8359

2.8386

2.8386

2.6578

3.0522

3.0522

9.1990

2.7294

3.7632

3.7632

3.0464

2.7189

3.4944

2.2232

2.5573

3.0612

3.7847

3.4337

2.6283

3.3264

2.2591

23.6546

23.6546

2.9943

2.2217

2.5414 \begin{tabular}{l|l|l|}
7 & 45 & $0.05 \%$ \\
\hline 11 & 97 & $0.07 \%$
\end{tabular}

\begin{tabular}{|r|r|r|}
\hline 11 & 97 & $0.07 \%$ \\
\hline 11 & 97 & $0.07 \%$ \\
\hline
\end{tabular}

\begin{tabular}{l|r|r|}
6 & 34 & $0.04 \%$ \\
\hline
\end{tabular}

\begin{tabular}{|r|r|r|}
\hline 6 & 34 & $0.04 \%$ \\
\hline 12 & 112 & $0.08 \%$ \\
\hline
\end{tabular}

\begin{tabular}{|r|r|r|}
\hline 12 & 112 & $0.08 \%$ \\
\hline 10 & 84 & $0.07 \%$ \\
\hline
\end{tabular}

\begin{tabular}{r|r|r|}
\hline 19 & 221 & $0.13 \%$ \\
\hline
\end{tabular}

\begin{tabular}{r|r|r|}
11 & 98 & $0.07 \%$ \\
5 & 24 & $0.03 \%$ \\
\hline
\end{tabular}

\begin{tabular}{r|r|r|}
5 & 24 & $0.03 \%$ \\
\hline 11 & 99 & $0.07 \%$ \\
\hline
\end{tabular}

\begin{tabular}{|r|r|r|}
\hline 11 & 99 & $0.07 \%$ \\
\hline 15 & 159 & $0.10 \%$ \\
\hline
\end{tabular}

\begin{tabular}{|r|r|r|}
15 & 159 & $0.10 \%$ \\
\hline 16 & 175 & $0.11 \%$ \\
\hline 17 & 191 & $0.11 \%$ \\
\hline
\end{tabular}

\begin{tabular}{l|l|l|}
17 & 191 & $0.11 \%$ \\
\hline
\end{tabular}

\begin{tabular}{|l|l|l|}
\hline 8 & 59 & $0.05 \%$ \\
\hline 9 & 72 & $0.06 \%$ \\
\hline
\end{tabular}

\begin{tabular}{|r|r|r|}
9 & 72 & $0.06 \%$ \\
\hline 14 & 144 & $0.09 \%$ \\
\hline
\end{tabular}

\begin{tabular}{|r|r|r|}
\hline 14 & 144 & $0.09 \%$ \\
\hline 11 & 100 & $0.07 \%$ \\
\hline 11 & 100 & $0.07 \%$
\end{tabular}

\begin{tabular}{r|r|r|}
\hline 11 & 100 & $0.07 \%$ \\
\hline 16 & 176 & $0.11 \%$ \\
\hline
\end{tabular}

\begin{tabular}{r|r|r|}
16 & 176 & $0.11 \%$ \\
\hline 5 & 25 & $0.03 \%$ \\
\hline
\end{tabular}

\begin{tabular}{l|l|l|}
13 & 130 & $0.09 \%$ \\
\hline
\end{tabular}

\begin{tabular}{r|r|r|}
\hline 3 & 8 & $0.02 \%$ \\
\hline 17 & 193 & $0.11 \%$ \\
\hline
\end{tabular}

\begin{tabular}{|r|r|r|}
\hline 17 & 193 & $0.11 \%$ \\
8 & 60 & $0.05 \%$ \\
\hline
\end{tabular}

\begin{tabular}{|l|l|r|}
\hline 12 & 116 & $0.08 \%$ \\
\hline 13 & 131 & $0.09 \%$ \\
\hline
\end{tabular}

\begin{tabular}{|r|r|r|}
\hline 17 & 131 & $0.09 \%$ \\
\hline 7 & 48 & $0.11 \%$ \\
\hline
\end{tabular}

\begin{tabular}{|l|l|l|}
7 & 48 & $0.05 \%$ \\
\hline 7 & 48 & $0.05 \%$ \\
\hline
\end{tabular}

\begin{tabular}{|r|r|r|}
\hline 11 & 102 & $0.07 \%$ \\
\hline 11 & 102 & $0.07 \%$ \\
\hline
\end{tabular}

\begin{tabular}{r|r|r|}
11 & 102 & $0.07 \%$ \\
\hline 10 & 88 & $0.07 \%$ \\
\hline
\end{tabular}

\begin{tabular}{r|r|r|}
\hline 10 & 88 & $0.07 \%$ \\
\hline 10 & 88 & $0.07 \%$ \\
\hline
\end{tabular}

\begin{tabular}{l|l|l|} 
& & \\
\hline 16 & 179 & $0.11 \%$ \\
\hline
\end{tabular}

\begin{tabular}{l|l|l|}
14 & 148 & $0.09 \%$ \\
\hline
\end{tabular}

\begin{tabular}{|r|r|r|}
\hline 17 & 196 & $0.11 \%$ \\
\hline
\end{tabular}

\begin{tabular}{|l|r|r|}
\hline 6 & 37 & $0.04 \%$ \\
\hline & 75 & $0.06 \%$ \\
\hline
\end{tabular}

\begin{tabular}{r|r|r|}
9 & 75 & $0.06 \%$ \\
\hline 9 & 75 & $0.06 \%$ \\
\hline
\end{tabular}

\begin{tabular}{|r|r|r|}
\hline 10 & 89 & $0.07 \%$ \\
\hline 8 & 62 & $0.05 \%$ \\
\hline
\end{tabular}

\begin{tabular}{|l|l|l|}
\hline 8 & 62 & $0.05 \%$ \\
\hline 8 & 62 & $0.05 \%$ \\
\hline
\end{tabular}

\begin{tabular}{r|r|r|}
\hline 7 & & \\
\hline 7 & 18 & $0.05 \%$ \\
\hline
\end{tabular}

\begin{tabular}{l|r|r|}
\hline 24 & 208 & $0.16 \%$ \\
\hline
\end{tabular}

\begin{tabular}{|r|r|r|}
\hline 14 & 88 & $0.09 \%$ \\
\hline 14 & 88 & $0.09 \%$ \\
\hline
\end{tabular}

\begin{tabular}{r|r|r|}
\hline 14 & 88 & $0.09 \%$ \\
\hline 17 & 132 & $0.11 \%$ \\
\hline
\end{tabular}

17

20

\begin{tabular}{|r|r|r|}
\hline 25 & 266 & $0.17 \%$ \\
\hline
\end{tabular}

\begin{tabular}{l|l|l|}
16 & 148 & $0.11 \%$ \\
\hline
\end{tabular}

\begin{tabular}{r|r|r|}
11 & 85 & $0.07 \%$ \\
\hline
\end{tabular}

\begin{tabular}{l|l|l|}
8 & 50 & $0.05 \%$ \\
\hline
\end{tabular}

\begin{tabular}{|r|r|r|}
\hline 9 & 62 & $0.06 \%$ \\
\hline
\end{tabular}

\begin{tabular}{|r|r|r|}
\hline 13 & 117 & $0.09 \%$ \\
\hline 9 & 64 & $0.06 \%$ \\
\hline
\end{tabular}

\begin{tabular}{|r|r|r|}
\hline 17 & 178 & $0.11 \%$ \\
\hline
\end{tabular}

\begin{tabular}{r|r}
2 & \\
2 & \\
10 &
\end{tabular}

\begin{tabular}{|l|l|}
\hline 2 & $0.01 \%$ \\
\hline 2 & $0.01 \%$ \\
\hline
\end{tabular}

\begin{tabular}{r|r|}
\hline 79 & $0.07 \%$ \\
\hline
\end{tabular}

\begin{tabular}{|l|l|}
\hline 181 & $0.11 \%$ \\
\hline
\end{tabular}

$0.09 \%$

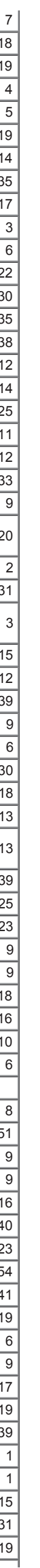




\begin{tabular}{|c|c|c|c|c|c|c|c|c|c|c|}
\hline MSigDB Cancer Neighborhood & Neighborhood of CASP10 & 2 & $4.07057 \mathrm{e}-6$ & $8.69066 \mathrm{e}-4$ & 2.1026 & 44 & 495 & $0.29 \%$ & 34 & 109 \\
\hline & Neighborhood of SMO & 15 & $1.00583 e-3$ & $2.86326 \mathrm{e}-2$ & 2.0900 & 22 & 249 & $0.15 \%$ & 16 & 51 \\
\hline & Neighborhood of DDX11 & 16 & $1.12707 \mathrm{e}-3$ & $3.00787 \mathrm{e}-2$ & 2.2648 & 18 & 188 & $0.12 \%$ & 13 & 38 \\
\hline & Neighborhood of AQP4 & 17 & $1.22872 \mathrm{e}-3$ & $3.08626 \mathrm{e}-2$ & 2.1924 & 19 & 205 & $0.13 \%$ & 14 & 43 \\
\hline & Neighborhood of RTN1 & 18 & $1.66154 \mathrm{e}-3$ & $3.94155 \mathrm{e}-2$ & 2.2465 & 17 & 179 & $0.11 \%$ & 11 & 44 \\
\hline & Neighborhood of JUND & 20 & $1.98422 \mathrm{e}-3$ & $4.23630 \mathrm{e}-2$ & 2.2095 & 17 & 182 & $0.11 \%$ & 15 & 67 \\
\hline \multirow[t]{5}{*}{ PANTHER Pathway } & TGF-beta signaling pathway & 1 & $3.26658 \mathrm{e}-6$ & $4.57321 \mathrm{e}-4$ & 2.0458 & 48 & 555 & $0.32 \%$ & 32 & 95 \\
\hline & Ras Pathway & 3 & $1.38449 \mathrm{e}-4$ & $6.46098 \mathrm{e}-3$ & 2.0119 & 33 & 388 & $0.22 \%$ & 22 & 70 \\
\hline & Beta2 adrenergic receptor signaling pathway & 14 & $2.56960 \mathrm{e}-3$ & $2.56959 \mathrm{e}-2$ & 2.0132 & 20 & 235 & $0.13 \%$ & 15 & 43 \\
\hline & Gamma-aminobutyric acid synthesis & 17 & $3.52648 \mathrm{e}-3$ & $2.90416 \mathrm{e}-2$ & 4.7309 & 5 & 25 & $0.03 \%$ & 3 & 5 \\
\hline & Transcription regulation by bZIP transcription factor & 19 & $4.72024 \mathrm{e}-3$ & $3.47807 \mathrm{e}-2$ & 2.0310 & 17 & 198 & $0.11 \%$ & 14 & 51 \\
\hline \multirow[t]{19}{*}{ Pathway Commons } & NCAM signaling for neurite out-growth & 3 & $1.05641 \mathrm{e}-5$ & $5.65883 \mathrm{e}-3$ & 2.2316 & 35 & 371 & $0.23 \%$ & 23 & 57 \\
\hline & Cell junction organization & 22 & $8.81267 e-5$ & $6.43726 \mathrm{e}-3$ & 2.0361 & 34 & 395 & $0.23 \%$ & 23 & 70 \\
\hline & $\mathrm{N}$-Glycan antennae elongation & 50 & $1.35988 \mathrm{e}-4$ & $4.37065 \mathrm{e}-3$ & 4.5296 & 9 & 47 & $0.06 \%$ & 4 & 9 \\
\hline & Tight junction interactions & 61 & $5.43390 \mathrm{e}-4$ & $1.43152 \mathrm{e}-2$ & 3.2525 & 11 & 80 & $0.07 \%$ & 6 & 19 \\
\hline & NCAM1 interactions & 63 & $5.96006 \mathrm{e}-4$ & $1.52029 \mathrm{e}-2$ & 2.3287 & 19 & 193 & $0.13 \%$ & 10 & 23 \\
\hline & Stimulation of the cell death response by PAK-2p34 & 68 & $7.27653 \mathrm{e}-4$ & $1.71962 \mathrm{e}-2$ & 6.5707 & 5 & 18 & $0.03 \%$ & 3 & 3 \\
\hline & Cell-cell junction organization & 70 & $9.52608 \mathrm{e}-4$ & $2.18692 \mathrm{e}-2$ & 2.1411 & 21 & 232 & $0.14 \%$ & 15 & 46 \\
\hline & Voltage gated Potassium channels & 72 & $1.00583 \mathrm{e}-3$ & $2.24496 \mathrm{e}-2$ & 2.0900 & 22 & 249 & $0.15 \%$ & 14 & 40 \\
\hline & Transport to the Golgi and subsequent modification & 73 & $1.01129 \mathrm{e}-3$ & $2.22622 \mathrm{e}-2$ & 2.7213 & 13 & 113 & $0.09 \%$ & 7 & 28 \\
\hline & $\mathrm{N}$-glycan antennae elongation in the medial/trans-Golgi & 74 & $1.01705 \mathrm{e}-3$ & $2.20865 \mathrm{e}-2$ & 3.4900 & 9 & 61 & $0.06 \%$ & 4 & 12 \\
\hline & CDO in myogenesis & 75 & $1.12707 \mathrm{e}-3$ & $2.41493 \mathrm{e}-2$ & 2.2648 & 18 & 188 & $0.12 \%$ & 12 & 24 \\
\hline & Myogenesis & 75 & $1.12707 e-3$ & $2.41493 \mathrm{e}-2$ & 2.2648 & 18 & 188 & $0.12 \%$ & 12 & 24 \\
\hline & Transcription of the HIV genome & 77 & $1.22872 \mathrm{e}-3$ & $2.56435 \mathrm{e}-2$ & 2.1924 & 19 & 205 & $0.13 \%$ & 14 & 61 \\
\hline & Ras signaling in the CD4+ TCR pathway & 78 & $1.30079 \mathrm{e}-3$ & $2.67996 \mathrm{e}-2$ & 2.1817 & 19 & 206 & $0.13 \%$ & 14 & 42 \\
\hline & Regulation of PAK-2p34 activity by PS-GAP/RHG10 & 79 & $1.37190 \mathrm{e}-3$ & $2.79069 \mathrm{e}-2$ & 11.8273 & 3 & 6 & $0.02 \%$ & 1 & 2 \\
\hline & p38MAPK events & 81 & $1.64552 \mathrm{e}-3$ & $3.26464 \mathrm{e}-2$ & 2.7034 & 12 & 105 & $0.08 \%$ & 7 & 9 \\
\hline & CD28 dependent Vav1 pathway & 82 & $1.75701 \mathrm{e}-3$ & $3.44330 \mathrm{e}-2$ & 2.8283 & 11 & 92 & $0.07 \%$ & 7 & 18 \\
\hline & Hormone ligand-binding receptors & 84 & $2.00028 \mathrm{e}-3$ & $3.82673 \mathrm{e}-2$ & 4.4352 & 6 & 32 & $0.04 \%$ & 3 & 10 \\
\hline & Signalling to RAS & 85 & $2.04608 \mathrm{e}-3$ & $3.86830 \mathrm{e}-2$ & 2.2663 & 16 & 167 & $0.11 \%$ & 11 & 21 \\
\hline \multirow{14}{*}{ MSigDB Pathway } & Genes related to PIP3 signaling in B lymphocytes & 5 & $5.42234 \mathrm{e}-5$ & $9.54331 \mathrm{e}-3$ & 3.1279 & 16 & 121 & $0.11 \%$ & 13 & 34 \\
\hline & Agrin in Postsynaptic Differentiation & 7 & $1.09022 \mathrm{e}-4$ & $1.37056 \mathrm{e}-2$ & 2.6594 & 19 & 169 & $0.13 \%$ & 12 & 36 \\
\hline & Genes involved in MyoGenessis & 11 & $2.39821 e-4$ & $1.91857 \mathrm{e}-2$ & 2.3232 & 22 & 224 & $0.15 \%$ & 15 & 29 \\
\hline & Fc Epsilon Receptor I Signaling in Mast Cells & 12 & $2.72454 \mathrm{e}-4$ & $1.99800 \mathrm{e}-2$ & 2.4137 & 20 & 196 & $0.13 \%$ & 14 & 38 \\
\hline & $\begin{array}{l}\text { Angiotensin II mediated activation of JNK Pathway via } \\
\text { Pyk2 dependent signaling }\end{array}$ & 15 & $4.91425 e-4$ & $2.88303 e-2$ & 2.3655 & 19 & 190 & $0.13 \%$ & 13 & 33 \\
\hline & Genes involved in Signaling by EGFR & 18 & $5.22447 \mathrm{e}-4$ & $2.55418 \mathrm{e}-2$ & 2.1958 & 22 & 237 & $0.15 \%$ & 17 & 49 \\
\hline & Genes involved in Signalling to RAS & 23 & $8.06999 \mathrm{e}-4$ & $3.08765 \mathrm{e}-2$ & 2.1692 & 21 & 229 & $0.14 \%$ & 13 & 26 \\
\hline & Links between Pyk2 and Map Kinases & 25 & $9.45191 \mathrm{e}-4$ & $3.32707 \mathrm{e}-2$ & 2.3655 & 17 & 170 & $0.11 \%$ & 12 & 28 \\
\hline & Genes related to chemotaxis & 25 & $9.45191 \mathrm{e}-4$ & $3.32707 e-2$ & 2.3655 & 17 & 170 & $0.11 \%$ & 14 & 45 \\
\hline & FAS signaling pathway ( CD95) & 28 & $1.27913 e-3$ & $4.02013 e-2$ & 2.7829 & 12 & 102 & $0.08 \%$ & 8 & 30 \\
\hline & Genes involved in Signalling to ERKs & 30 & $1.66461 \mathrm{e}-3$ & $4.88285 \mathrm{e}-2$ & 2.0093 & 22 & 259 & $0.15 \%$ & 14 & 34 \\
\hline & p38 MAPK Signaling Pathway & 31 & $1.70112 \mathrm{e}-3$ & $4.82899 \mathrm{e}-2$ & 2.1835 & 18 & 195 & $0.12 \%$ & 14 & 41 \\
\hline & Genes involved in Activation of Rac & 32 & $1.79720 \mathrm{e}-3$ & $4.94229 \mathrm{e}-2$ & 3.2256 & 9 & 66 & $0.06 \%$ & 6 & 14 \\
\hline & JNK MAPK Pathway & 33 & $1.87129 \mathrm{e}-3$ & $4.99010 \mathrm{e}-2$ & 2.2217 & 17 & 181 & $0.11 \%$ & 13 & 38 \\
\hline \multirow[t]{12}{*}{ MGI Expression: Detected } & TS9_extraembryonic component & 254 & $3.23539 \mathrm{e}-8$ & $1.06335 \mathrm{e}-6$ & 2.0761 & 66 & 752 & $0.44 \%$ & 44 & 133 \\
\hline & TS23_lens & 260 & $3.98160 \mathrm{e}-8$ & $1.27840 \mathrm{e}-6$ & 2.2151 & 56 & 598 & $0.37 \%$ & 33 & 122 \\
\hline & TS28_cerebral cortex layer; layer VI & 306 & $2.68037 \mathrm{e}-7$ & $7.31234 \mathrm{e}-6$ & 2.5442 & 37 & 344 & $0.25 \%$ & 20 & 54 \\
\hline & TS28_tectum & 314 & $3.62226 \mathrm{e}-7$ & $9.63012 e-6$ & 2.2794 & 45 & 467 & $0.30 \%$ & 25 & 68 \\
\hline & TS28_cerebral cortex layer; layer III & 321 & $4.59460 \mathrm{e}-7$ & $1.19488 \mathrm{e}-5$ & 2.1746 & 49 & 533 & $0.33 \%$ & 30 & 97 \\
\hline & TS28_cerebral cortex layer; layer II & 339 & $1.27556 \mathrm{e}-6$ & $3.14111 \mathrm{e}-5$ & 2.1768 & 45 & 489 & $0.30 \%$ & 27 & 80 \\
\hline & $\begin{array}{l}\text { TS17_tail; nervous system; central nervous system; } \\
\text { future spinal cord; neural tube }\end{array}$ & 346 & $1.63082 \mathrm{e}-6$ & $3.93470 e-5$ & 3.0197 & 24 & 188 & $0.16 \%$ & 10 & 19 \\
\hline & TS17_optic cup & 357 & $2.31149 \mathrm{e}-6$ & $5.40514 e-5$ & 2.0066 & 52 & 613 & $0.35 \%$ & 35 & 90 \\
\hline & TS28_cerebral cortex layer; layer V & 369 & $3.10165 e-6$ & $7.01696 \mathrm{e}-5$ & 2.2159 & 40 & 427 & $0.27 \%$ & 23 & 78 \\
\hline & TS28_cerebral cortex layer; layer IV & 378 & $3.68109 \mathrm{e}-6$ & $8.12956 e-5$ & 2.4497 & 32 & 309 & $0.21 \%$ & 20 & 56 \\
\hline & TS21_arm & 392 & $5.29916 \mathrm{e}-6$ & $1.12850 \mathrm{e}-4$ & 2.8244 & 24 & 201 & $0.16 \%$ & 12 & 26 \\
\hline & TS17_tail; nervous system; central nervous system & 397 & $5.77378 \mathrm{e}-6$ & $1.21409 \mathrm{e}-4$ & 2.8104 & 24 & 202 & $0.16 \%$ & 10 & 25 \\
\hline
\end{tabular}


|TS17_tail; nervous system; central nervous system; future spinal cord

TS17_tail; nervous system

TS25_cerebral cortex; intermediate zone

TS20_pons

TS21_neural retinal epithelium

TS15_tail; mesenchyme

TS18_pancreas primordium

TS17 atrio-ventricular canal \begin{tabular}{|l|l|l|}
397 & $5.77378 \mathrm{e}-6$ & $1.21409 \mathrm{e}-4$ \\
\hline
\end{tabular}

\begin{tabular}{|r|r|r|}
\hline 400 & $6.28676 \mathrm{e}-6$ & $1.31205 \mathrm{e}-4$ \\
\hline
\end{tabular}

\begin{tabular}{|l|l|l|}
406 & $7.29506 \mathrm{e}-6$ & $1.49998 \mathrm{e}-4$ \\
\hline
\end{tabular}

\begin{tabular}{|l|l|l|}
\hline 409 & $7.59796 \mathrm{e}-6$ & $1.55080 \mathrm{e}-4$ \\
\hline
\end{tabular}

\begin{tabular}{|l|l|l|}
\hline 414 & $8.65362 \mathrm{e}-6$ & $1.74494 \mathrm{e}-4$ \\
\hline
\end{tabular}

\begin{tabular}{l|l|l|l|}
\hline 415 & $9.19265 \mathrm{e}-6$ & $1.84916 \mathrm{e}-4$ \\
\hline
\end{tabular}

\begin{tabular}{|l|l|l|}
\hline 421 & $1.14689 \mathrm{e}-5$ & $2.27417 \mathrm{e}-4$ \\
\hline 429 & $1.27079 \mathrm{e}-5$ & $2.47285 \mathrm{e}-4$ \\
\hline
\end{tabular}

Genes down-regulated and displaying decreased copy number in glioblastoma samples.

Genes down-regulated in Caco-2 cells (intestinal

epithelium) after coculture with the probiotic bacteria L.

casei for $6 \mathrm{~h}$.

Selected gradually up-regulated genes whose expression profile follows that of HEMGN [Gene $I D=55363]$ in the TLX1 [Gene ID=3195] Tet On iEBHX15-4 cells (pro-erythroblasts).

Genes from the blue module which are up-regulated in

HAEC cells (primary aortic endothelium) after exposure

to the oxidized 1-palmitoyl-2-arachidonyl-sn-3-

glycerophosphorylcholine (oxPAPC).

Genes from the turquoise module which are upregulated in HAEC cells (primary aortic endothelium)

after exposure to the oxidized 1-palmitoyl-2arachidonyl-sn-3-glycerophosphocholine (oxPAPC).

Selected gradually up-regulated genes whose expression profile follows that of HBZ [Gene ID=3050]

in the TLX1 [Gene ID=3195] Tet On iEBHX15-4 cells (pro-erythroblasts).

Genes up-regulated in metaplastic carcinoma of the breast (MCB) subclass 2 compared to the MCB subclass 1.

Genes consistently up-regulated in HMEC cells (primary mammary epithelium) upon expression of TERT [Gene ID=7015] off a retroviral vector.

Genes commonly up-regulated in CD-1 and CD-2 clusters of multiple myeloma samples and which were higher expressed in the CD-1 group.

Genes up-regulated in HCT8/S11 cells (colon cancer) engineered to stably express NTN1 [Gene ID=1630] off a plasmid vector.

Down-regulated in ovarian epithelial cells (MCV152) 72 hours following FSH treatment, compared to untreated

Genes down-regulated in poorly differentiated thyroid carcinoma (PDTC) compared to anaplastic thyroid carcinoma (ATC).

Genes up-regulated in metastatic breast cancer tumors having type 2 amplification in the 20q13 region; involves MYBL2, STK6 and ZNF217 [Gene ID=4605, 6790, 7764]

Genes up-regulated in MCF10A cells (breast cancer) by expression of LSM1 [Gene ID=27257] off a letiviral vector.

Genes whose expression peaked at 60 min after stimulation of MCF10A cells with EGF [Gene ID=1950]. Genes that cooperate with MYC [Gene ID=4609] and TBX2 [Gene ID=6909] to transform MEF cells (embryo fibroblasts).

Genes down-regulated in A549 cells (lung cancer) expressing MIRLET7A3 [Gene ID=406883] microRNA off a plasmid vector.

Genes up-regulated in MCF-7 cells (breast cancer) by selective estrogen receptor modulators (SERM) 4-

hydroxytamoxifen, raloxifene, or ICI 182780 but not by estradiol [PubChem=44959, 5035, 3478439, 5757].

Amplification hot spot 25: colocalized fragile sites and cancer genes in the 2q13-q36 region.

Top genes up-regulated in subcutaneous tumors from highly metastatic pancreatic cancer cells.

Motif DGATADGAHWAGATA matches EVI1: ecotropic viral integration site 1

Motif NNAACAATNN matches SOX5: SRY (sex

determining region $\mathrm{Y}$ )-box 5

\begin{tabular}{r|r|r|r}
\hline & & & \\
\hline 9 & $1.45423 \mathrm{e}-7$ & $3.83594 \mathrm{e}-5$ &
\end{tabular}

\begin{tabular}{r|r|r|}
9 & $1.45423 e-7$ & $3.83594 \mathrm{e}-5$ \\
\hline 15 & $6.81669 \mathrm{e}-7$ & $1.07885 \mathrm{e}-4$
\end{tabular}

\begin{tabular}{|l|l|l|l|}
\hline 30 & $1.19598 \mathrm{e}-5$ & $9.46420 \mathrm{e}-4$ & \\
\hline
\end{tabular}

37

37

\begin{tabular}{|l|l|}
\hline 65 \\
\hline 78 \\
\hline
\end{tabular}

4.8522

\begin{tabular}{|r|r|r|r|r}
15 & $6.81669 \mathrm{e}-7$ & $1.07885 \mathrm{e}-4$ & 2.0919 & \\
\hline 26 & $8.15062 \mathrm{e}-6$ & $7.44215 \mathrm{e}-4$ & 2.9924 & \\
\hline
\end{tabular}

\begin{tabular}{|l|l|l|l|l|}
34 & $1.92442 \mathrm{e}-5$ & $1.34370 \mathrm{e}-3$ & 2.1974 & \\
\hline & & & & \\
\hline
\end{tabular}

\begin{tabular}{r|r|r|r|r}
37 & $2.25585 \mathrm{e}-5$ & $1.44740 \mathrm{e}-3$ & 2.5923 & \\
\hline 43 & $4.56966 \mathrm{e}-5$ & $2.52288 \mathrm{e}-3$ & 2.6020 & \\
\hline 53 & $1.01940 \mathrm{e}-4$ & $4.56615 \mathrm{e}-3$ & 2.0207 & \\
\hline
\end{tabular}

\begin{tabular}{|c|c|c|c|}
\hline 64 & $1.95376 \mathrm{e}-4$ & $7.24724 \mathrm{e}-3$ & 2.1432 \\
\hline 65 & $2.00486 e-4$ & $7.32238 \mathrm{e}-3$ & 2.8035 \\
\hline 72 & $2.72828 e-4$ & $8.99574 e-3$ & 2.8386 \\
\hline 78 & $3.61178 \mathrm{e}-4$ & $1.09928 \mathrm{e}-2$ & 2.0317 \\
\hline
\end{tabular}

\begin{tabular}{|c|c|c|c|c|c|c|c|c|}
\hline 78 & $3.61178 \mathrm{e}-4$ & $1.09928 \mathrm{e}-2$ & 2.0317 & 28 & 326 & $0.19 \%$ & 20 & 69 \\
\hline 81 & $3.98184 \mathrm{e}-4$ & $1.16702 \mathrm{e}-2$ & 2.0193 & 28 & 328 & $0.19 \%$ & 20 & 106 \\
\hline 83 & $4.58474 \mathrm{e}-4$ & $1.31135 \mathrm{e}-2$ & 2.3191 & 20 & 204 & $0.13 \%$ & 13 & 40 \\
\hline 89 & $5.10502 \mathrm{e}-4$ & $1.36172 \mathrm{e}-2$ & 2.4977 & 17 & 161 & $0.11 \%$ & 12 & 36 \\
\hline 96 & $7.45735 e-4$ & $1.84414 \mathrm{e}-2$ & 3.1349 & 11 & 83 & $0.07 \%$ & 7 & 11 \\
\hline 111 & $1.34133 e-3$ & $2.86876 \mathrm{e}-2$ & 2.0076 & 23 & 271 & $0.15 \%$ & 19 & 77 \\
\hline 120 & $1.64532 \mathrm{e}-3$ & $3.25500 \mathrm{e}-2$ & 3.5705 & 8 & 53 & $0.05 \%$ & 7 & 19 \\
\hline 122 & $2.00132 \mathrm{e}-3$ & $3.89437 \mathrm{e}-2$ & 2.9568 & 10 & 80 & $0.07 \%$ & 5 & 9 \\
\hline 126 & $2.32791 \mathrm{e}-3$ & $4.38609 \mathrm{e}-2$ & 2.0304 & 20 & 233 & $0.13 \%$ & 11 & 42 \\
\hline 7 & $5.04266 e-12$ & $4.43034 \mathrm{e}-10$ & 2.3594 & 78 & 782 & $0.52 \%$ & 54 & 186 \\
\hline 9 & $9.85693 e-12$ & $6.73557 \mathrm{e}-10$ & 2.2477 & 84 & 884 & $0.56 \%$ & 57 & 191 \\
\hline
\end{tabular}

\begin{tabular}{|c|c|c|c|c|c|c|c|c|}
\hline 78 & $3.61178 \mathrm{e}-4$ & $1.09928 \mathrm{e}-2$ & 2.0317 & 28 & 326 & $0.19 \%$ & 20 & 69 \\
\hline 81 & $3.98184 \mathrm{e}-4$ & $1.16702 \mathrm{e}-2$ & 2.0193 & 28 & 328 & $0.19 \%$ & 20 & 106 \\
\hline 83 & $4.58474 \mathrm{e}-4$ & $1.31135 \mathrm{e}-2$ & 2.3191 & 20 & 204 & $0.13 \%$ & 13 & 40 \\
\hline 89 & $5.10502 \mathrm{e}-4$ & $1.36172 \mathrm{e}-2$ & 2.4977 & 17 & 161 & $0.11 \%$ & 12 & 36 \\
\hline 96 & $7.45735 \mathrm{e}-4$ & $1.84414 \mathrm{e}-2$ & 3.1349 & 11 & 83 & $0.07 \%$ & 7 & 11 \\
\hline 111 & $1.34133 \mathrm{e}-3$ & $2.86876 \mathrm{e}-2$ & 2.0076 & 23 & 271 & $0.15 \%$ & 19 & 77 \\
\hline 120 & $1.64532 \mathrm{e}-3$ & $3.25500 \mathrm{e}-2$ & 3.5705 & 8 & 53 & $0.05 \%$ & 7 & 19 \\
\hline 122 & $2.00132 \mathrm{e}-3$ & $3.89437 \mathrm{e}-2$ & 2.9568 & 10 & 80 & $0.07 \%$ & 5 & 9 \\
\hline 126 & $2.32791 \mathrm{e}-3$ & $4.38609 \mathrm{e}-2$ & 2.0304 & 20 & 233 & $0.13 \%$ & 11 & 42 \\
\hline 7 & $5.04266 e-12$ & $4.43034 \mathrm{e}-10$ & 2.3594 & 78 & 782 & $0.52 \%$ & 54 & 186 \\
\hline 9 & $9.85693 e-12$ & $6.73557 e-10$ & 2.2477 & 84 & 884 & $0.56 \%$ & 57 & 191 \\
\hline
\end{tabular}


Motif YNGTTNNNATT (no known TF)

Motif TGATTTRY matches GFI1: growth factor

independent 1

Motif NNNNNGTAAATAAACA matches FOXC1:

forkhead box C1

Motif NNNNAACAATRGNN matches SOX9: SRY (sex

determining region Y)-box 9 (campomelic dysplasia,

autosomal sex-reversal)

Motif NAWTGTTTRTTT matches FOXD3: forkhead

box D3

Motif NNNNNNNWATGCAAATNNNWNNA matches

POU2F1: POU domain, class 2, transcription factor

Motif NNNTGTTTATNTR matches FOXJ1: forkhead

box J1

Motif KGNANTRTTTRYTTW matches FOXA2

forkhead box A2

Motif CAGNYGKNAAA (no known TF)

Motif NWATAAGTATWT matches NKX3-1: NK3

transcription factor related, locus 1 (Drosophila)

Motif AGATAAGATAN matches EVI1: ecotropic viral

integration site 1

Motif TGCNHNCWYCCYCATTAKTNNDCNMNHYCN

matches HOXA5: homeobox A5

Motif TGACAGKTTTAYGA matches MEIS1: Meis1,

myeloid ecotropic viral integration site 1 homolog

(mouse)\&lt;br\&gt; HOXA9: homeobox A9

Motif CCYCNRRSTNGCGTGASA matches AHR: aryl

hydrocarbon receptor

Motif CCTGAGAATAATC matches ALX4: aristaless-

like homeobox 4

Motif CRGAARNNNNCGA (no known TF)

Motif NNNTNNNGNGTGANN matches PAX8: paired

box gene 8

Motif GCGSCMNTTT (no known TF)

\section{Targets of MicroRNA TTGCACT,MIR-130A,MIR-}

301, MIR-130B

Targets of MicroRNA TAGCTTT,MIR-9

Targets of MicroRNA TTGGAGA,MIR-515-5P,MIR-

$519 \mathrm{E}$

Targets of MicroRNA GACAGGG,MIR-339

Targets of MicroRNA CTCTGGA,MIR-520A,MIR-525

Targets of MicroRNA CAGCCTC,MIR-485-5P

Targets of MicroRNA AACATTC,MIR-409-3P

Targets of MicroRNA GTACAGG,MIR-486

Targets of MicroRNA CCATCCA,MIR-432

Targets of MicroRNA AAGGGAT,MIR-188

Targets of MicroRNA ATCATGA,MIR-433

Targets of MicroRNA TCTGATA,MIR-361

Targets of MicroRNA ATTACAT,MIR-380-3P

Targets of MicroRNA GCAAGAC,MIR-431

Targets of MicroRNA AGGAGTG,MIR-483

Targets of MicroRNA CGGTGTG,MIR-220

Targets of MicroRNA CACGTTT,MIR-302A

Targets of MicroRNA TCTAGAG,MIR-517

Targets of MicroRNA TACGGGT,MIR-99A,MIR-

100,MIR-99B

Targets of MicroRNA GTCAACC,MIR-380-5P

Molybdopterin synthase/thiamin biosynthesis sulphur

carrier, beta-grasp

Homeodomain protein CUT

K Homology

Lambda repressor-like, DNA-binding

Pyridoxal phosphate-dependent decarboxylase

Ubiquitin-related modifier 1

LCCL

Zinc finger and BTB domain-containing protein 24 CUX2/ONECUT2/ONECUT1/CUX1/ONECUT3

Mucin-17 precursor

\begin{tabular}{|c|c|c|c|c|c|c|c|c|}
\hline 10 & \begin{tabular}{|l|l}
$1.43755 e-11$ \\
\end{tabular} & $8.84090 \mathrm{e}-10$ & 2.0614 & 101 & 1159 & $0.67 \%$ & 66 & 272 \\
\hline 11 & $1.54359 \mathrm{e}-10$ & $8.63005 e-9$ & 2.0957 & 87 & 982 & $0.58 \%$ & 63 & 233 \\
\hline 14 & $2.08805 e-10$ & $9.17251 \mathrm{e}-9$ & 2.0929 & 86 & 972 & $0.57 \%$ & 61 & 191 \\
\hline 17 & $4.17455 \mathrm{e}-10$ & $1.51021 \mathrm{e}-8$ & 2.1738 & 76 & 827 & $0.51 \%$ & 56 & 181 \\
\hline 20 & $2.29658 \mathrm{e}-9$ & $7.06199 \mathrm{e}-8$ & 2.1396 & 72 & 796 & $0.48 \%$ & 51 & 164 \\
\hline 22 & $5.16741 \mathrm{e}-9$ & $1.44453 e-7$ & 2.1363 & 69 & 764 & $0.46 \%$ & 50 & 192 \\
\hline 24 & $8.15527 \mathrm{e}-9$ & $2.08979 \mathrm{e}-7$ & 2.0521 & 74 & 853 & $0.49 \%$ & 44 & 170 \\
\hline 29 & $2.98044 \mathrm{e}-8$ & $6.32058 \mathrm{e}-7$ & 2.0317 & 70 & 815 & $0.47 \%$ & 42 & 170 \\
\hline 31 & $4.56150 \mathrm{e}-8$ & $9.04942 \mathrm{e}-7$ & 2.9236 & 33 & 267 & $0.22 \%$ & 21 & 54 \\
\hline 32 & $1.03760 \mathrm{e}-7$ & $1.99413 e-6$ & 2.0762 & 61 & 695 & $0.41 \%$ & 49 & 177 \\
\hline 53 & $1.01001 \mathrm{e}-6$ & $1.17199 \mathrm{e}-5$ & 2.0485 & 53 & 612 & $0.35 \%$ & 35 & 147 \\
\hline 90 & $1.05074 \mathrm{e}-5$ & $7.18002 e-5$ & 2.8593 & 22 & 182 & $0.15 \%$ & 15 & 31 \\
\hline 94 & $1.39560 e-5$ & $9.13080 e-5$ & 2.1014 & 39 & 439 & $0.26 \%$ & 25 & 92 \\
\hline 109 & $2.88204 \mathrm{e}-5$ & $1.62610 \mathrm{e}-4$ & 2.3175 & 29 & 296 & $0.19 \%$ & 16 & 65 \\
\hline 187 & $4.87106 \mathrm{e}-4$ & $1.60198 \mathrm{e}-3$ & 3.2937 & 11 & 79 & $0.07 \%$ & 6 & 13 \\
\hline 283 & $4.06047 \mathrm{e}-3$ & $8.82400 \mathrm{e}-3$ & 2.1768 & 15 & 163 & $0.10 \%$ & 15 & 35 \\
\hline 285 & $4.08169 \mathrm{e}-3$ & $8.80787 \mathrm{e}-3$ & 2.1144 & 16 & 179 & $0.11 \%$ & 8 & 26 \\
\hline 324 & $7.74008 \mathrm{e}-3$ & $1.46918 \mathrm{e}-2$ & 2.0275 & 15 & 175 & $0.10 \%$ & 10 & 49 \\
\hline 1 & $9.08124 \mathrm{e}-13$ & $2.00695 \mathrm{e}-10$ & 2.0072 & 121 & 1426 & $0.81 \%$ & 82 & 328 \\
\hline 7 & $9.57895 \mathrm{e}-9$ & $3.02421 \mathrm{e}-7$ & 2.0669 & 72 & 824 & $0.48 \%$ & 53 & 196 \\
\hline 9 & $1.71559 \mathrm{e}-8$ & $4.21274 \mathrm{e}-7$ & 2.3748 & 51 & 508 & $0.34 \%$ & 36 & 121 \\
\hline 11 & $2.71086 \mathrm{e}-8$ & $5.44636 \mathrm{e}-7$ & 2.8291 & 36 & 301 & $0.24 \%$ & 21 & 53 \\
\hline 13 & $6.92849 \mathrm{e}-8$ & $1.17784 \mathrm{e}-6$ & 2.1003 & 61 & 687 & $0.41 \%$ & 40 & 131 \\
\hline 20 & $9.15782 \mathrm{e}-7$ & $1.01194 \mathrm{e}-5$ & 2.0552 & 53 & 610 & $0.35 \%$ & 39 & 116 \\
\hline 21 & $1.24821 \mathrm{e}-6$ & $1.31360 \mathrm{e}-5$ & 2.2004 & 44 & 473 & $0.29 \%$ & 30 & 117 \\
\hline 35 & $5.57217 \mathrm{e}-6$ & $3.51843 e-5$ & 2.9737 & 22 & 175 & $0.15 \%$ & 14 & 48 \\
\hline 40 & $8.90288 \mathrm{e}-6$ & $4.91884 \mathrm{e}-5$ & 2.6171 & 26 & 235 & $0.17 \%$ & 15 & 51 \\
\hline 41 & $9.18552 \mathrm{e}-6$ & $4.95122 \mathrm{e}-5$ & 2.3808 & 31 & 308 & $0.21 \%$ & 24 & 65 \\
\hline 48 & $3.11490 \mathrm{e}-5$ & $1.43415 \mathrm{e}-4$ & 2.1744 & 33 & 359 & $0.22 \%$ & 23 & 92 \\
\hline 49 & $3.49919 \mathrm{e}-5$ & $1.57820 \mathrm{e}-4$ & 2.3743 & 27 & 269 & $0.18 \%$ & 19 & 75 \\
\hline 55 & $7.40156 e-5$ & $2.97408 \mathrm{e}-4$ & 2.2729 & 27 & 281 & $0.18 \%$ & 20 & 85 \\
\hline 71 & $4.10532 \mathrm{e}-4$ & $1.27785 \mathrm{e}-3$ & 2.5451 & 17 & 158 & $0.11 \%$ & 12 & 36 \\
\hline 72 & $4.54822 \mathrm{e}-4$ & $1.39605 \mathrm{e}-3$ & 2.0970 & 25 & 282 & $0.17 \%$ & 16 & 59 \\
\hline 80 & $1.45010 \mathrm{e}-3$ & $4.00591 \mathrm{e}-3$ & 3.6392 & 8 & 52 & $0.05 \%$ & 5 & 6 \\
\hline 89 & $2.47288 \mathrm{e}-3$ & $6.14053 e-3$ & 2.7104 & 11 & 96 & $0.07 \%$ & 10 & 24 \\
\hline 102 & $6.19342 \mathrm{e}-3$ & $1.34191 \mathrm{e}-2$ & 2.0239 & 16 & 187 & $0.11 \%$ & 10 & 37 \\
\hline 111 & $9.85266 \mathrm{e}-3$ & $1.96165 \mathrm{e}-2$ & 2.1668 & 12 & 131 & $0.08 \%$ & 9 & 20 \\
\hline 123 & $1.72653 \mathrm{e}-2$ & $3.10213 e-2$ & 2.4261 & 8 & 78 & $0.05 \%$ & 7 & 19 \\
\hline 8 & $1.54242 \mathrm{e}-5$ & $1.53239 \mathrm{e}-2$ & 18.9237 & 4 & 5 & $0.03 \%$ & 2 & 2 \\
\hline 9 & $1.61096 \mathrm{e}-5$ & $1.42266 \mathrm{e}-2$ & 4.3670 & 12 & 65 & $0.08 \%$ & 6 & 7 \\
\hline 10 & $1.68831 \mathrm{e}-5$ & $1.34187 \mathrm{e}-2$ & 2.8549 & 21 & 174 & $0.14 \%$ & 12 & 39 \\
\hline 11 & $3.35775 \mathrm{e}-5$ & $2.42613 e-2$ & 2.6551 & 22 & 196 & $0.15 \%$ & 12 & 28 \\
\hline 13 & $5.90158 \mathrm{e}-5$ & $3.60813 \mathrm{e}-2$ & 6.6233 & 7 & 25 & $0.05 \%$ & 4 & 8 \\
\hline 14 & $7.55387 \mathrm{e}-5$ & $4.28844 \mathrm{e}-2$ & 23.6546 & 3 & 3 & $0.02 \%$ & 1 & 1 \\
\hline 15 & $8.32193 e-5$ & $4.40951 \mathrm{e}-2$ & 9.8561 & 5 & 12 & $0.03 \%$ & 3 & 6 \\
\hline 1 & $2.63778 \mathrm{e}-6$ & $2.10732 \mathrm{e}-2$ & 16.8961 & 5 & 7 & $0.03 \%$ & 1 & 1 \\
\hline 2 & $4.05736 \mathrm{e}-6$ & $1.62071 \mathrm{e}-2$ & 5.4208 & 11 & 48 & $0.07 \%$ & 5 & 5 \\
\hline 3 & $1.09606 \mathrm{e}-5$ & $2.91880 \mathrm{e}-2$ & 7.0088 & 8 & 27 & $0.05 \%$ & 1 & 1 \\
\hline
\end{tabular}


GREAT Table Output: Genomic Regions Enrichment of Annotations Tool, Bejerano Lab, Stanford University

\begin{tabular}{|c|c|c|c|c|c|c|c|c|c|c|}
\hline & Zinc finger and BTB domain-containing protein & 4 & $1.65725 e-5$ & $3.30995 e-2$ & 7.8849 & 7 & 21 & $0.05 \%$ & 4 & 4 \\
\hline HGNC Gene Families & CUT & 1 & $1.61096 \mathrm{e}-5$ & $7.90981 \mathrm{e}-3$ & 4.3670 & 12 & 65 & $0.08 \%$ & 6 & 7 \\
\hline & MUC & 2 & $1.69869 e-5$ & $4.17029 \mathrm{e}-3$ & 3.8065 & 14 & 87 & $0.09 \%$ & 3 & 16 \\
\hline & ZBTB & 3 & $8.61125 \mathrm{e}-5$ & $1.40938 \mathrm{e}-2$ & 2.2151 & 28 & 299 & $0.19 \%$ & 16 & 41 \\
\hline
\end{tabular}

The test set contains 14,977 (4\%) of all 354,275 regions.

GO Molecular Function has 3,402 terms covering 14,878 (84\%) of all 17,635 genes.

The test set picked 2,666 genes, the background set picked 14,129 genes.

GO Biological Process has 8,762 terms covering 14,731 (84\%) of all 17,635 genes.

3,402 ontology terms were tested $(100 \%)$ using an annotation count range of [1, Inf].

GO Cellular Component has 1,121 terms covering 16,374 (93\%) of all 17,635 genes. 8,762 ontology terms were tested $(100 \%)$ using an annotation count range of [1, Inf].

Mouse Phenotype has 7,294 terms covering 6,429 (36\%) of all 17,635 genes.

Disease Ontology has 2,234 terms covering 7,911 (45\%) of all 17,635 genes.

MSigDB Cancer Neighborhood has 427 terms covering 4,385 (25\%) of all 17,635 genes.

PANTHER Pathway has 140 terms covering 2,183 (12\%) of all 17,635 genes.

Pathway Commons has 1,607 terms covering 6,072 (34\%) of all 17,635 genes. 1,121 ontology terms were tested $(100 \%)$ using an annotation count range of [1, Inf]. 7,294 ontology terms were tested $(100 \%)$ using an annotation count range of [1, Inf]. 2,234 ontology terms were tested $(100 \%)$ using an annotation count range of [1, Inf]. 427 ontology terms were tested $(100 \%)$ using an annotation count range of [1, Inf].

MSigDB Pathway has 880 terms covering 6,686 (38\%) of all 17,635 genes. 140 ontology terms were tested (100\%) using an annotation count range of [1, Inf]. 1,607 ontology terms were tested $(100 \%)$ using an annotation count range of [1, Inf] 880 ontology terms were tested $(100 \%)$ using an annotation count range of [1, Inf] MGI Expression: Detected has 8,348 terms covering 11,006 (62\%) of all 17,635 genes. MSigDB Perturbation has 2,374 terms covering 16,270 (92\%) of all 17,635 genes. 8,348 ontology terms were tested $(100 \%)$ using an annotation count range of [1, Inf]. MSigDB Predicted Promoter Motifs has 615 terms covering 8,958 (51\%) of all 17,635 genes. 2,374 ontology terms were tested $(100 \%)$ using an annotation count range of [1, Inf] MSigDB miRNA Motifs has 221 terms covering 6,031 (34\%) of all 17,635 genes. 615 ontology terms were tested $(100 \%)$ using an annotation count range of [1, Inf]. 221 ontology terms were tested (100\%) using an annotation count range of [1, Inf]. InterPro has 7,948 terms covering 16,411 (93\%) of all 17,635 genes.

TreeFam has 7,989 terms covering 16,523 (94\%) of all 17,635 genes. 7,948 ontology terms were tested $(100 \%)$ using an annotation count range of [1, Inf]. HGNC Gene Families has 491 terms covering 6,911 (39\%) of all 17,635 genes. 7,989 ontology terms were tested (100\%) using an annotation count range of [1, Inf] GREAT version 2.0.2 491 ontology terms were tested (100\%) using an annotation count range of [1, Inf]

Species assembly: hg18

Association rule: Basal+extension: 5000 bp upstream, 5000 bp downstream, 0 bp max extension, curated regulatory domains included 